\title{
Statistical inference for critical continuous state and continuous time branching processes with immigration
}

\author{
Mátyás Barczy ${ }^{*, \diamond}$, Kristóf KÖRMendi* ${ }^{* *}$ Gyula PaP***
}

* Faculty of Informatics, University of Debrecen, Pf. 12, H-4010 Debrecen, Hungary.

** MTA-SZTE Analysis and Stochastics Research Group, Bolyai Institute, University of Szeged, Aradi vértanúk tere 1, H-6720 Szeged, Hungary.

*** Bolyai Institute, University of Szeged, Aradi vértanúk tere 1, H-6720 Szeged, Hungary.

e-mails: barczy.matyas@inf.unideb.hu (M. Barczy), kormendi@math.u-szeged.hu (K. Körmendi), papgy@math.u-szeged.hu (G. Pap).

$\diamond$ Corresponding author.

\begin{abstract}
We study asymptotic behavior of conditional least squares estimators for critical continuous state and continuous time branching processes with immigration based on discrete time (low frequency) observations.
\end{abstract}

\section{$\|$ \\ 1 Introduction}

Under some mild moment condition (see (2.3)), a continuous state and continuous time branching process with immigration (CBI process) can be represented as a pathwise unique strong solution of the stochastic differential equation (SDE)

$$
\begin{aligned}
X_{t}= & X_{0}+\int_{0}^{t}\left(\beta+\widetilde{b} X_{s}\right) \mathrm{d} s+\int_{0}^{t} \sqrt{2 c \max \left\{0, X_{s}\right\}} \mathrm{d} W_{s} \\
& +\int_{0}^{t} \int_{0}^{\infty} \int_{0}^{\infty} z \mathbb{1}_{\left\{u \leqslant X_{s-}\right\}} \tilde{N}(\mathrm{~d} s, \mathrm{~d} z, \mathrm{~d} u)+\int_{0}^{t} \int_{0}^{\infty} z M(\mathrm{~d} s, \mathrm{~d} z)
\end{aligned}
$$

for $t \in[0, \infty)$, where $\beta, c \in[0, \infty), \widetilde{b} \in \mathbb{R}$, and $\left(W_{t}\right)_{t \geqslant 0}$ is a standard Wiener process, $N$ and $M$ are Poisson random measures on $(0, \infty)^{3}$ and on $(0, \infty)^{2}$ with intensity measures $\mathrm{d} s \mu(\mathrm{d} z) \mathrm{d} u$ and $\mathrm{d} s \nu(\mathrm{d} z)$, respectively, $\tilde{N}(\mathrm{~d} s, \mathrm{~d} z, \mathrm{~d} u):=N(\mathrm{~d} s, \mathrm{~d} z, \mathrm{~d} u)-\mathrm{d} s \mu(\mathrm{d} z) \mathrm{d} u$ is the compensated Poisson random measure corresponding to $N$, the branching jump measure $\mu$ and the immigration jump measure $\nu$ satisfy some moment conditions, and $\left(W_{t}\right)_{t \geqslant 0}, \quad N$ and $M$ are independent, see Dawson and Li [8, Theorems 5.1 and 5.2]. The model is called subcritical, critical or supercritical if $\widetilde{b}<0, \widetilde{b}=0$ or $\widetilde{b}>0$, see Huang et al. [10, page 1105].

2010 Mathematics Subject Classifications: 62F12, 60J80.

Key words and phrases: branching processes with immigration, conditional least squares estimator. 
Based on discrete time (low frequency) observations $\left(X_{k}\right)_{k \in\{0,1, \ldots, n\}}, \quad n \in\{1,2, \ldots\}$, Huang et al. [10] derived weighted conditional least squares (CLS) estimator of $(\widetilde{b}, \beta)$. Under some additional moment conditions, they showed the following results: in the subcritical case the estimator of $(\widetilde{b}, \beta)$ is asymptotically normal; in the critical case the estimator of $\widetilde{b}$ has a non-normal limit, but the asymptotic behavior of the estimator of $\beta$ remained open; in the supercritical case the estimator of $\widetilde{b}$ is asymptotically normal with a random scaling, but the estimator of $\beta$ is not weakly consistent.

Overbeck and Rydén [19] considered CLS and weighted CLS estimators for the well-known Cox-Ingersoll-Ross model, which is, in fact, a diffusion CBI process (without jump part), i.e., when $\mu=0$ and $\nu=0$ in (1.1). Based on discrete time observations $\left(X_{k}\right)_{k \in\{0,1, \ldots, n\}}$, $n \in\{1,2, \ldots\}$, they derived CLS estimator of $(\widetilde{b}, \beta, c)$ and proved its asymptotic normality in the subcritical case. Note that Li and Ma [18] started to investigate the asymptotic behaviour of the CLS and weighted CLS estimators of the parameters $(\widetilde{b}, \beta)$ in the subcritical case for a Cox-Ingersoll-Ross model driven by a stable noise, which is again a special CBI process (with jump part).

For simplicity, we suppose $X_{0}=0$. We suppose that $c, \mu$ and $\nu$ are known, and we derive the CLS estimator of $(\widetilde{b}, \widetilde{\beta})$ based on discrete time (low frequency) observations $\left(X_{k}\right)_{k \in\{1, \ldots, n\}}, \quad n \in\{1,2, \ldots\}$, where $\widetilde{\beta}:=\beta+\int_{0}^{\infty} z \nu(\mathrm{d} z)$. In the critical case, i.e, when $\widetilde{b}=0$, under some moment conditions, we describe the asymptotic behavior of these CLS estimators as $n \rightarrow \infty$, provided that $\beta \neq 0$ or $\nu \neq 0$, see Theorem 3.1 . We point out that the limit distributions are non-normal in general. In the present paper we do not investigate the asymptotic behavior of CLS estimators of $(\widetilde{b}, \widetilde{\beta})$ in the subcritical and supercritical cases, it could be the topic of separate papers.

\section{CBI processes}

Let $\mathbb{Z}_{+}, \mathbb{N}, \mathbb{R}, \mathbb{R}_{+}$and $\mathbb{R}_{++}$denote the set of non-negative integers, positive integers, real numbers, non-negative real numbers and positive real numbers, respectively. For $x, y \in \mathbb{R}$, we will use the notations $x \wedge y:=\min \{x, y\}$ and $x^{+}:=\max \{0, x\}$. By $\|\boldsymbol{x}\|$ and $\|\boldsymbol{A}\|$, we denote the Euclidean norm of a vector $\boldsymbol{x} \in \mathbb{R}^{d}$ and the induced matrix norm of a matrix $\boldsymbol{A} \in \mathbb{R}^{d \times d}$, respectively. The null vector and the null matrix will be denoted by $\mathbf{0}$. By $C_{\mathrm{c}}^{2}\left(\mathbb{R}_{+}, \mathbb{R}\right)$ we denote the set of twice continuously differentiable real-valued functions on $\mathbb{R}_{+}$ with compact support. Convergence in distribution and in probability will be denoted by $\stackrel{\mathcal{D}}{\longrightarrow}$ and $\stackrel{\mathbb{P}}{\longrightarrow}$, respectively.

2.1 Definition. A tuple $(c, \beta, b, \nu, \mu)$ is called a set of admissible parameters if $c, \beta \in \mathbb{R}_{+}$, $b \in \mathbb{R}$, and $\nu$ and $\mu$ are Borel measures on $(0, \infty)$ satisfying $\int_{0}^{\infty}(1 \wedge z) \nu(\mathrm{d} z)<\infty$ and $\int_{0}^{\infty}\left(z \wedge z^{2}\right) \mu(\mathrm{d} z)<\infty$.

2.2 Theorem. Let $(c, \beta, b, \nu, \mu)$ be a set of admissible parameters. Then there exists a unique conservative transition semigroup $\left(P_{t}\right)_{t \in \mathbb{R}_{+}}$acting on the Banach space (endowed with the 
supremum norm) of real-valued bounded Borel-measurable functions on the state space $\mathbb{R}_{+}$ such that its infinitesimal generator is

$$
\begin{aligned}
(\mathcal{A} f)(x)= & c x f^{\prime \prime}(x)+(\beta+b x) f^{\prime}(x)+\int_{0}^{\infty}(f(x+z)-f(x)) \nu(\mathrm{d} z) \\
& +x \int_{0}^{\infty}\left(f(x+z)-f(x)-f^{\prime}(x)(1 \wedge z)\right) \mu(\mathrm{d} z)
\end{aligned}
$$

for $f \in C_{\mathrm{c}}^{2}\left(\mathbb{R}_{+}, \mathbb{R}\right)$ and $x \in \mathbb{R}_{+}$. Moreover, the Laplace transform of the transition semigroup $\left(P_{t}\right)_{t \in \mathbb{R}_{+}}$has a representation

$$
\int_{0}^{\infty} \mathrm{e}^{-\lambda y} P_{t}(x, \mathrm{~d} y)=\mathrm{e}^{-x v(t, \lambda)-\int_{0}^{t} \psi(v(s, \lambda)) \mathrm{d} s}, \quad x \in \mathbb{R}_{+}, \quad \lambda \in \mathbb{R}_{+}, \quad t \in \mathbb{R}_{+},
$$

where, for any $\lambda \in \mathbb{R}_{+}$, the continuously differentiable function $\mathbb{R}_{+} \ni t \mapsto v(t, \lambda) \in \mathbb{R}_{+}$is the unique locally bounded solution to the differential equation

$$
\partial_{t} v(t, \lambda)=-\varphi(v(t, \lambda)), \quad v(0, \lambda)=\lambda,
$$

with

$$
\varphi(\lambda):=c \lambda^{2}-b \lambda+\int_{0}^{\infty}\left(\mathrm{e}^{-\lambda z}-1+\lambda(1 \wedge z)\right) \mu(\mathrm{d} z), \quad \lambda \in \mathbb{R}_{+},
$$

and

$$
\psi(\lambda):=\beta \lambda+\int_{0}^{\infty}\left(1-\mathrm{e}^{-\lambda z}\right) \nu(\mathrm{d} z), \quad \lambda \in \mathbb{R}_{+}
$$

2.3 Remark. This theorem is a special case of Theorem 2.7 of Duffie et al. [9] with $m=1$, $n=0$ and zero killing rate. The unique existence of a locally bounded solution to the differential equation (2.2) is proved by $\mathrm{Li}$ [17, page 45]. Here, we point out that the moment condition on $\mu$ given in Definition 2.1 (which is stronger than the one (2.11) in Definition 2.6 in Duffie et al. [9]) ensures that the semigroup $\left(P_{t}\right)_{t \in \mathbb{R}_{+}}$is conservative (we do not need the one-point compactification of $\mathbb{R}_{+}^{d}$ ), see Duffie et al. [9, Lemma 9.2] and Li [17, page 45]. For the continuity of the function $\mathbb{R}_{+} \times \mathbb{R}_{+} \ni(t, \lambda) \mapsto v(t, \lambda)$, see Duffie et al. [9, Proposition 6.4]. Finally, we note that the infinitesimal generator (2.1) can be rewritten in another equivalent form

$$
\begin{aligned}
(\mathcal{A} f)(x)= & c x f^{\prime \prime}(x)+\left(\beta+\left(b+\int_{1}^{\infty}(z-1) \mu(\mathrm{d} z)\right) x\right) f^{\prime}(x) \\
& +\int_{0}^{\infty}(f(x+z)-f(x)) \nu(\mathrm{d} z)+x \int_{0}^{\infty}\left(f(x+z)-f(x)-z f^{\prime}(x)\right) \mu(\mathrm{d} z),
\end{aligned}
$$

where $b+\int_{1}^{\infty}(z-1) \mu(\mathrm{d} z)$ is nothing else but $\widetilde{b}$ given in (2.5).

2.4 Definition. A conservative Markov process with state space $\mathbb{R}_{+}$and with transition semigroup $\left(P_{t}\right)_{t \in \mathbb{R}_{+}}$given in Theorem 2.2 is called a CBI process with parameters $(c, \beta, b, \nu, \mu)$. The function $\mathbb{R}_{+} \ni \lambda \mapsto \varphi(\lambda) \in \mathbb{R}$ is called its branching mechanism, and the function $\mathbb{R}_{+} \ni \lambda \mapsto \psi(\lambda) \in \mathbb{R}_{+}$is called its immigration mechanism. 
Note that the branching mechanism depends only on the parameters $c, b$ and $\mu$, while the immigration mechanism depends only on the parameters $\beta$ and $\nu$.

Let $\left(X_{t}\right)_{t \in \mathbb{R}_{+}}$be a CBI process with parameters $(c, \beta, b, \nu, \mu)$ such that $\mathbb{E}\left(X_{0}\right)<\infty$ and the moment condition

$$
\int_{1}^{\infty} z \nu(\mathrm{d} z)<\infty
$$

holds. Then, by formula (3.4) in Barczy et al. [5],

$$
\mathbb{E}\left(X_{t} \mid X_{0}=x\right)=\mathrm{e}^{\widetilde{b} t} x+\widetilde{\beta} \int_{0}^{t} \mathrm{e}^{\widetilde{b} u} \mathrm{~d} u, \quad x \in \mathbb{R}_{+}, \quad t \in \mathbb{R}_{+},
$$

where

$$
\widetilde{b}:=b+\int_{1}^{\infty}(z-1) \mu(\mathrm{d} z), \quad \widetilde{\beta}:=\beta+\int_{0}^{\infty} z \nu(\mathrm{d} z) .
$$

Note that $\widetilde{b} \in \mathbb{R}$ and $\widetilde{\beta} \in \mathbb{R}_{+}$due to (2.3). One can give probabilistic interpretations of the modified parameters $\widetilde{b}$ and $\widetilde{\beta}$, namely, $\mathrm{e}^{\widetilde{b}}=\mathbb{E}\left(Y_{1} \mid Y_{0}=1\right)$ and $\widetilde{\beta}=\mathbb{E}\left(Z_{1} \mid Z_{0}=0\right)$, where $\left(Y_{t}\right)_{t \in \mathbb{R}_{+}}$and $\left(Z_{t}\right)_{t \in \mathbb{R}_{+}}$are CBI processes with parameters $(c, 0, b, 0, \mu)$ and $(0, \beta, 0, \nu, 0)$, respectively, see formula (2.4). The processes $\left(Y_{t}\right)_{t \in \mathbb{R}_{+}}$and $\left(Z_{t}\right)_{t \in \mathbb{R}_{+}}$can be considered as pure branching (without immigration) and pure immigration (without branching) processes, respectively. Consequently, $\mathrm{e}^{\widetilde{b}}$ and $\widetilde{\beta}$ may be called the branching and immigration mean, respectively. Moreover, by the help of the modified parameters $\widetilde{b}$ and $\widetilde{\beta}$, the SDE (1.1) can be rewritten as

$$
\begin{aligned}
X_{t}= & X_{0}+\int_{0}^{t}\left(\widetilde{\beta}+\widetilde{b} X_{s}\right) \mathrm{d} s+\int_{0}^{t} \sqrt{2 c X_{s}^{+}} \mathrm{d} W_{s} \\
& +\int_{0}^{t} \int_{0}^{\infty} \int_{0}^{\infty} z \mathbb{1}_{\left\{u \leqslant X_{s-}\right\}} \widetilde{N}(\mathrm{~d} s, \mathrm{~d} z, \mathrm{~d} u)+\int_{0}^{t} \int_{0}^{\infty} z \widetilde{M}(\mathrm{~d} s, \mathrm{~d} z)
\end{aligned}
$$

for $t \in[0, \infty)$, where $\widetilde{M}(\mathrm{~d} s, \mathrm{~d} z):=M(\mathrm{~d} s, \mathrm{~d} z)-\mathrm{d} s \mu(\mathrm{d} z)$.

Next we will recall a convergence result for critical CBI processes.

A function $f: \mathbb{R}_{+} \rightarrow \mathbb{R}$ is called càdlàg if it is right continuous with left limits. Let $\mathbb{D}\left(\mathbb{R}_{+}, \mathbb{R}\right)$ and $\mathbb{C}\left(\mathbb{R}_{+}, \mathbb{R}\right)$ denote the space of all $\mathbb{R}$-valued càdlàg and continuous functions on $\mathbb{R}_{+}$, respectively. Let $\mathcal{D}_{\infty}\left(\mathbb{R}_{+}, \mathbb{R}\right)$ denote the Borel $\sigma$-field in $\mathbb{D}\left(\mathbb{R}_{+}, \mathbb{R}\right)$ for the metric characterized by Jacod and Shiryaev [14, VI.1.15] (with this metric $\mathbb{D}\left(\mathbb{R}_{+}, \mathbb{R}\right.$ ) is a complete and separable metric space). For $\mathbb{R}$-valued stochastic processes $\left(\mathcal{Y}_{t}\right)_{t \in \mathbb{R}_{+}}$and $\left(\mathcal{Y}_{t}^{(n)}\right)_{t \in \mathbb{R}_{+}}$, $n \in \mathbb{N}$, with càdlàg paths we write $\mathcal{Y}^{(n)} \stackrel{\mathcal{D}}{\longrightarrow} \mathcal{Y}$ as $n \rightarrow \infty$ if the distribution of $\mathcal{Y}^{(n)}$ on the space $\left(\mathbb{D}\left(\mathbb{R}_{+}, \mathbb{R}\right), \mathcal{D}_{\infty}\left(\mathbb{R}_{+}, \mathbb{R}\right)\right)$ converges weakly to the distribution of $\mathcal{Y}$ on the space $\left(\mathbb{D}\left(\mathbb{R}_{+}, \mathbb{R}\right), \mathcal{D}_{\infty}\left(\mathbb{R}_{+}, \mathbb{R}\right)\right)$ as $n \rightarrow \infty$. Concerning the notation $\stackrel{\mathcal{D}}{\longrightarrow}$ we note that if $\xi$ and $\xi_{n}, n \in \mathbb{N}$, are random elements with values in a metric space $(E, \rho)$, then we also denote by $\xi_{n} \stackrel{\mathcal{D}}{\longrightarrow} \xi$ the weak convergence of the distributions of $\xi_{n}$ on the space $(E, \mathcal{B}(E))$ towards 
the distribution of $\xi$ on the space $(E, \mathcal{B}(E))$ as $n \rightarrow \infty$, where $\mathcal{B}(E)$ denotes the Borel $\sigma$-algebra on $E$ induced by the given metric $\rho$.

The following convergence theorem can be found in Huang et al. [10, Theorem 2.3].

2.5 Theorem. Let $\left(X_{t}\right)_{t \in \mathbb{R}_{+}}$be a CBI process with parameters $(c, \beta, b, \nu, \mu)$ such that $X_{0}=$ 0 , the moment conditions

$$
\int_{1}^{\infty} z^{q} \nu(\mathrm{d} \boldsymbol{z})<\infty, \quad \int_{1}^{\infty} z^{q} \mu(\mathrm{d} \boldsymbol{z})<\infty
$$

hold with $q=2$, and $\widetilde{b}=0$ (hence the process is critical). Then

$$
\left(\mathcal{X}_{t}^{(n)}\right)_{t \in \mathbb{R}_{+}}:=\left(n^{-1} X_{\lfloor n t\rfloor}\right)_{t \in \mathbb{R}_{+}} \stackrel{\mathcal{D}}{\longrightarrow}\left(\mathcal{Y}_{t}\right)_{t \in \mathbb{R}_{+}} \quad \text { as } n \rightarrow \infty
$$

in $\mathbb{D}\left(\mathbb{R}_{+}, \mathbb{R}\right)$, where $\left(\mathcal{Y}_{t}\right)_{t \in \mathbb{R}_{+}}$is the pathwise unique strong solution of the $S D E$

$$
\mathrm{d} \mathcal{Y}_{t}=\widetilde{\beta} \mathrm{d} t+\sqrt{C \mathcal{Y}_{t}^{+}} \mathrm{d} \mathcal{W}_{t}, \quad t \in \mathbb{R}_{+}, \quad \mathcal{Y}_{0}=0
$$

where $\left(\mathcal{W}_{t}\right)_{t \in \mathbb{R}_{+}}$is a standard Brownian motion and

$$
C:=2 c+\int_{0}^{\infty} z^{2} \mu(\mathrm{d} z) \in \mathbb{R}_{+}
$$

2.6 Remark. The SDE (2.9) has a pathwise unique strong solution $\left(\mathcal{Y}_{t}^{(y)}\right)_{t \in \mathbb{R}_{+}}$for all initial values $\mathcal{Y}_{0}^{(y)}=y \in \mathbb{R}$, and if the initial value $y$ is nonnegative, then $\mathcal{Y}_{t}^{(y)}$ is nonnegative for all $t \in \mathbb{R}_{+}$with probability one, since $\widetilde{\beta} \in \mathbb{R}_{+}$, see, e.g., Ikeda and Watanabe [11, Chapter IV, Example 8.2].

2.7 Remark. Note that $C=0$ if and only if $c=0$ and $\mu=0$, when the pathwise unique strong solution of (2.9) is the deterministic function $\mathcal{Y}_{t}=\widetilde{\beta} t, \quad t \in \mathbb{R}_{+}$. Further, $C=\operatorname{Var}\left(Y_{1} \mid Y_{0}=1\right)$, see Proposition B.3, where $\left(Y_{t}\right)_{t \in \mathbb{R}_{+}}$is a pure branching CBI process with parameters $(c, 0, b, 0, \mu)$. Clearly, $C$ depends only on the branching mechanism.

\section{Main results}

Let $\left(X_{t}\right)_{t \in \mathbb{R}_{+}}$be a CBI process with parameters $(c, \beta, b, \nu, \mu)$ such that the moment condition (2.3) holds. For the sake of simplicity, we suppose $X_{0}=0$. In the sequel we also assume that $\beta \neq 0$ or $\nu \neq 0$ (i.e., the immigration mechanism is non-zero), equivalently, $\widetilde{\beta} \neq 0$ (where $\widetilde{\beta}$ is defined in (2.5) ), otherwise $X_{t}=0$ for all $t \in \mathbb{R}_{+}$, following from (2.4). The parameter $\widetilde{b}$ can also be called the criticality parameter, since $\left(X_{t}\right)_{t \in \mathbb{R}_{+}}$is critical if and only if $\widetilde{b}=0$.

For $k \in \mathbb{Z}_{+}$, let $\mathcal{F}_{k}:=\sigma\left(X_{0}, X_{1}, \ldots, X_{k}\right)$. Since $\left(X_{k}\right)_{k \in \mathbb{Z}_{+}}$is a time-homogeneous Markov process, by (2.4),

$$
\mathbb{E}\left(X_{k} \mid \mathcal{F}_{k-1}\right)=\mathbb{E}\left(X_{k} \mid X_{k-1}\right)=\varrho X_{k-1}+\bar{\beta}, \quad k \in \mathbb{N},
$$


where

$$
\varrho:=\mathrm{e}^{\widetilde{b}} \in \mathbb{R}_{++}, \quad \bar{\beta}:=\widetilde{\beta} \int_{0}^{1} \mathrm{e}^{\widetilde{b} s} \mathrm{~d} s \in \mathbb{R}_{+} .
$$

Note that $\bar{\beta}=\mathbb{E}\left(X_{1} \mid X_{0}=0\right)$, see (2.4). Note also that $\bar{\beta}$ depends both on the branching and immigration mechanisms, although $\widetilde{\beta}$ depends only on the immigration mechanism. Let us introduce the sequence

$$
M_{k}:=X_{k}-\mathbb{E}\left(X_{k} \mid \mathcal{F}_{k-1}\right)=X_{k}-\varrho X_{k-1}-\bar{\beta}, \quad k \in \mathbb{N},
$$

of martingale differences with respect to the filtration $\left(\mathcal{F}_{k}\right)_{k \in \mathbb{Z}_{+}}$. By $(\underline{3.3})$, the process $\left(X_{k}\right)_{k \in \mathbb{Z}_{+}}$ satisfies the recursion

$$
X_{k}=\varrho X_{k-1}+\bar{\beta}+M_{k}, \quad k \in \mathbb{N} \text {. }
$$

For each $n \in \mathbb{N}$, a CLS estimator $\left(\widehat{\varrho}_{n}, \widehat{\bar{\beta}}_{n}\right)$ of $(\varrho, \bar{\beta})$ based on a sample $X_{1}, \ldots, X_{n}$ can be obtained by minimizing the sum of squares

$$
\sum_{k=1}^{n}\left(X_{k}-\varrho X_{k-1}-\bar{\beta}\right)^{2}
$$

with respect to $(\varrho, \bar{\beta})$ over $\mathbb{R}^{2}$, and it has the form

$$
\left[\begin{array}{l}
\widehat{\varrho}_{n} \\
\widehat{\bar{\beta}}_{n}
\end{array}\right]:=\frac{1}{n \sum_{k=1}^{n} X_{k-1}^{2}-\left(\sum_{k=1}^{n} X_{k-1}\right)^{2}}\left[\begin{array}{c}
n \sum_{k=1}^{n} X_{k} X_{k-1}-\sum_{k=1}^{n} X_{k} \sum_{k=1}^{n} X_{k-1} \\
\sum_{k=1}^{n} X_{k} \sum_{k=1}^{n} X_{k-1}^{2}-\sum_{k=1}^{n} X_{k} X_{k-1} \sum_{k=1}^{n} X_{k-1}
\end{array}\right]
$$

on the set

$$
H_{n}:=\left\{\omega \in \Omega: n \sum_{k=1}^{n} X_{k-1}^{2}(\omega)-\left(\sum_{k=1}^{n} X_{k-1}(\omega)\right)^{2}>0\right\},
$$

see, e.g., Wei and Winnicki [21, formulas (1.4), (1.5)]. In the sequel we investigate the critical case. By Lemma C.1, $\mathbb{P}\left(H_{n}\right) \rightarrow 1$ as $n \rightarrow \infty$. Let us introduce the function $h: \mathbb{R}^{2} \rightarrow \mathbb{R}_{++} \times \mathbb{R}$ by

$$
h(\widetilde{b}, \widetilde{\beta}):=\left(\mathrm{e}^{\widetilde{b}}, \widetilde{\beta} \int_{0}^{1} \mathrm{e}^{\widetilde{b} s} \mathrm{~d} s\right)=(\varrho, \bar{\beta}), \quad(\widetilde{b}, \widetilde{\beta}) \in \mathbb{R}^{2} .
$$

Note that $h$ is bijective having inverse

$$
h^{-1}(\varrho, \bar{\beta})=\left(\log (\varrho), \frac{\bar{\beta}}{\int_{0}^{1} \varrho^{s} \mathrm{~d} s}\right)=(\widetilde{b}, \widetilde{\beta}), \quad(\varrho, \bar{\beta}) \in \mathbb{R}_{++} \times \mathbb{R} .
$$

Theorem 3.4 will imply that the CLS estimator $\widehat{\varrho}_{n}$ of $\varrho$ is weakly consistent, hence, for sufficiently large $n \in \mathbb{N}$ with probability converging to 1 , $\left(\widehat{\varrho}_{n}, \widehat{\bar{\beta}}_{n}\right)$ falls into the set $\mathbb{R}_{++} \times \mathbb{R}$, and hence

$$
\left(\widehat{\varrho}_{n}, \widehat{\bar{\beta}}_{n}\right)=\arg \min (\varrho, \bar{\beta}) \in \mathbb{R}_{++} \times \mathbb{R} \sum_{k=1}^{n}\left(X_{k}-\varrho X_{k-1}-\bar{\beta}\right)^{2} .
$$


Thus one can introduce a natural estimator of $(\widetilde{b}, \widetilde{\beta})$ by applying the inverse of $h$ to the CLS estimator of $(\varrho, \bar{\beta})$, that is,

$$
\left.\widehat{\widetilde{b}}_{n}, \widehat{\widetilde{\beta}}_{n}\right):=h^{-1}\left(\widehat{\varrho}_{n}, \widehat{\bar{\beta}}_{n}\right)=\left(\log \left(\widehat{\varrho}_{n}\right), \frac{\widehat{\bar{\beta}}_{n}}{\int_{0}^{1}\left(\widehat{\varrho}_{n}\right)^{s} \mathrm{~d} s}\right), \quad n \in \mathbb{N},
$$

on the set $\left\{\omega \in \Omega:\left(\widehat{\varrho}_{n}(\omega), \widehat{\bar{\beta}}_{n}(\omega)\right) \in \mathbb{R}_{++} \times \mathbb{R}\right\}$. We also obtain

$$
\left(\widehat{\widetilde{b}}_{n}, \widehat{\widetilde{\beta}}_{n}\right)=\arg \min _{(\widetilde{b}, \widetilde{\beta}) \in \mathbb{R}^{2}} \sum_{k=1}^{n}\left(X_{k}-\mathrm{e}^{\widetilde{b}} X_{k-1}-\widetilde{\beta} \int_{0}^{1} \mathrm{e}^{\widetilde{b} s} \mathrm{~d} s\right)^{2}
$$

for sufficiently large $n \in \mathbb{N}$ with probability converging to 1 , hence $\left(\widehat{\widetilde{b}}_{n}, \widehat{\widetilde{\beta}}_{n}\right)$ is the CLS estimator of $(\widetilde{b}, \widetilde{\beta})$ for sufficiently large $n \in \mathbb{N}$ with probability converging to 1 . We would like to stress the point that the estimator $\left(\widehat{\widetilde{b}}_{n}, \widehat{\widetilde{\beta}}_{n}\right)$ exists only for sufficiently large $n \in \mathbb{N}$ with probability converging to 1 . However, as all our results are asymptotic, this will not cause a problem.

3.1 Theorem. Let $\left(X_{t}\right)_{t \in \mathbb{R}_{+}}$be a CBI process with parameters $(c, \beta, b, \nu, \mu)$ such that $X_{0}=$ 0 , the moment conditions (2.7) hold with $q=8, \beta \neq 0$ or $\nu \neq 0$, and $\widetilde{b}=0$ (hence the process is critical). Then the probability of the existence of the estimator $\left(\widehat{\widetilde{b}}_{n}, \widehat{\widetilde{\beta}}_{n}\right)$ converges to 1 as $n \rightarrow \infty$ and

$$
\left[\begin{array}{c}
n\left(\widehat{\widetilde{b}}_{n}-\widetilde{b}\right) \\
\widehat{\widetilde{\beta}}_{n}-\widetilde{\beta}
\end{array}\right] \stackrel{\mathcal{D}}{\longrightarrow} \frac{1}{\int_{0}^{1} \mathcal{Y}_{t}^{2} \mathrm{~d} t-\left(\int_{0}^{1} \mathcal{Y}_{t} \mathrm{~d} t\right)^{2}}\left[\begin{array}{c}
\int_{0}^{1} \mathcal{Y}_{t} \mathrm{~d} \mathcal{M}_{t}-\mathcal{M}_{1} \int_{0}^{1} \mathcal{Y}_{t} \mathrm{~d} t \\
\mathcal{M}_{1} \int_{0}^{1} \mathcal{Y}_{t}^{2} \mathrm{~d} t-\int_{0}^{1} \mathcal{Y}_{t} \mathrm{~d} t \int_{0}^{1} \mathcal{Y}_{t} \mathrm{~d} \mathcal{M}_{t}
\end{array}\right]
$$

as $n \rightarrow \infty$, where $\left(\mathcal{Y}_{t}\right)_{t \in \mathbb{R}_{+}}$is the pathwise unique strong solution of the SDE (2.9), and $\mathcal{M}_{t}:=\mathcal{Y}_{t}-\widetilde{\beta} t, \quad t \in \mathbb{R}_{+}$.

If, in addition, $c=0$ and $\mu=0$ (hence the process is a pure immigration process), then

$$
\left[\begin{array}{c}
n^{3 / 2} \underset{\left(\widehat{\widetilde{b}}_{n}-\widetilde{b}\right)}{n^{1 / 2}\left(\widehat{\widetilde{\beta}}_{n}-\widetilde{\beta}\right)}
\end{array}\right] \stackrel{\mathcal{D}}{\longrightarrow} \mathcal{N}_{2}\left(\mathbf{0}, \int_{0}^{\infty} z^{2} \nu(\mathrm{d} z)\left[\begin{array}{cc}
\frac{1}{3}(\widetilde{\beta})^{2} & \frac{1}{2} \widetilde{\beta} \\
\frac{1}{2} \widetilde{\beta} & 1
\end{array}\right]^{-1}\right) \quad \text { as } n \rightarrow \infty
$$

3.2 Remark. By Remark 2.7, if $C=0$, then $\mathcal{M}_{t}=0, \quad t \in \mathbb{R}_{+}$, further, by (3.7), $n\left(\widehat{\widetilde{b}}_{n}-\widetilde{b}\right) \stackrel{\mathcal{D}}{\longrightarrow} 0$ and $\widehat{\widetilde{\beta}}_{n}-\widetilde{\beta} \stackrel{\mathcal{D}}{\longrightarrow} 0$ as $n \rightarrow \infty$.

3.3 Remark. If $C \neq 0$ then the estimator $\widehat{\widetilde{\beta}}_{n}$ is not consistent. The same holds for the discrete time analogues of $\widetilde{\beta}$, for instance, the immigration mean of a critical Galton-Watson branching process with immigration, see Wei and Winnicki [22], or the innovation mean of a positive regular unstable INAR(2) process, see Barczy et al. [4].

Theorem 3.1 will follow from the following statement. 
3.4 Theorem. Under the assumptions of Theorem 3.1, the probability of the existence of unique CLS estimator $\left(\widehat{\varrho}_{n}, \widehat{\bar{\beta}}_{n}\right)$ converges to 1 as $n \rightarrow \infty$ and

$$
\left[\begin{array}{c}
n\left(\widehat{\varrho}_{n}-\varrho\right) \\
\widehat{\bar{\beta}}_{n}-\bar{\beta}
\end{array}\right] \stackrel{\mathcal{D}}{\longrightarrow} \frac{1}{\int_{0}^{1} \mathcal{Y}_{t}^{2} \mathrm{~d} t-\left(\int_{0}^{1} \mathcal{Y}_{t} \mathrm{~d} t\right)^{2}}\left[\begin{array}{c}
\int_{0}^{1} \mathcal{Y}_{t} \mathrm{~d} \mathcal{M}_{t}-\mathcal{M}_{1} \int_{0}^{1} \mathcal{Y}_{t} \mathrm{~d} t \\
\mathcal{M}_{1} \int_{0}^{1} \mathcal{Y}_{t}^{2} \mathrm{~d} t-\int_{0}^{1} \mathcal{Y}_{t} \mathrm{~d} t \int_{0}^{1} \mathcal{Y}_{t} \mathrm{~d} \mathcal{M}_{t}
\end{array}\right]
$$

as $n \rightarrow \infty$.

If, in addition, $c=0$ and $\mu=0$ (hence the process is a pure immigration process), then

$$
\left[\begin{array}{c}
n^{3 / 2}\left(\widehat{\varrho}_{n}-\varrho\right) \\
n^{1 / 2}\left(\widehat{\bar{\beta}}_{n}-\bar{\beta}\right)
\end{array}\right] \stackrel{\mathcal{D}}{\longrightarrow} \mathcal{N}_{2}\left(\mathbf{0}, \int_{0}^{\infty} z^{2} \nu(\mathrm{d} z)\left[\begin{array}{cc}
\frac{1}{3}(\widetilde{\beta})^{2} & \frac{1}{2} \widetilde{\beta} \\
\frac{1}{2} \widetilde{\beta} & 1
\end{array}\right]^{-1}\right) \quad \text { as } n \rightarrow \infty \text {. }
$$

Proof of Theorem 3.1. Before Theorem 3.1 we have already investigated the existence of $\left(\widehat{\widetilde{b}}_{n}, \widehat{\widetilde{\beta}}_{n}\right)$. Now we apply Lemma D.1 with $S=T=\mathbb{R}^{2}, C=\mathbb{R}^{2}$,

$$
\begin{gathered}
\xi_{n}=\left[\begin{array}{c}
n\left(\widehat{\varrho}_{n}-\varrho\right) \\
\widehat{\bar{\beta}}_{n}-\bar{\beta}
\end{array}\right]=\left[\begin{array}{c}
n\left(\widehat{\varrho}_{n}-1\right) \\
\widehat{\bar{\beta}}_{n}-\widetilde{\beta}
\end{array}\right], \\
\xi=\frac{1}{\int_{0}^{1} \mathcal{Y}_{t}^{2} \mathrm{~d} t-\left(\int_{0}^{1} \mathcal{Y}_{t} \mathrm{~d} t\right)^{2}}\left[\begin{array}{c}
\int_{0}^{1} \mathcal{Y}_{t} \mathrm{~d} \mathcal{M}_{t}-\mathcal{M}_{1} \int_{0}^{1} \mathcal{Y}_{t} \mathrm{~d} t \\
\mathcal{M}_{1} \int_{0}^{1} \mathcal{Y}_{t}^{2} \mathrm{~d} t-\int_{0}^{1} \mathcal{Y}_{t} \mathrm{~d} t \int_{0}^{1} \mathcal{Y}_{t} \mathrm{~d} \mathcal{M}_{t}
\end{array}\right],
\end{gathered}
$$

with functions $f: \mathbb{R}^{2} \rightarrow \mathbb{R}^{2}$ and $f_{n}: \mathbb{R}^{2} \rightarrow \mathbb{R}^{2}, n \in \mathbb{N}$, given by

$$
f\left(\left[\begin{array}{l}
x \\
y
\end{array}\right]\right):=\left[\begin{array}{l}
x \\
y
\end{array}\right], \quad(x, y) \in \mathbb{R}^{2}, \quad f_{n}\left(\left[\begin{array}{l}
x \\
y
\end{array}\right]\right):=\left[\begin{array}{c}
n \log \left(1+\frac{x}{n}\right) \\
\frac{y+\widetilde{\beta}}{\int_{0}^{1}\left(1+\frac{x}{n}\right)^{s} \mathrm{~d} s}-\widetilde{\beta}
\end{array}\right]
$$

for $(x, y) \in \mathbb{R}^{2}$ with $x>-n$, and $f_{n}(x, y):=0$ otherwise. We have $f_{n}\left(n\left(\widehat{\varrho}_{n}-1\right), \widehat{\bar{\beta}}_{n}-\widetilde{\beta}\right)=$ $\left(n\left(\widehat{\widetilde{b}}_{n}-\widetilde{b}\right), \widehat{\widetilde{\beta}}_{n}-\widetilde{\beta}\right)$ on the set $\left\{\omega \in \Omega: \widehat{\varrho}_{n}(\omega) \in \mathbb{R}_{++}\right\}$, and $f_{n}\left(x_{n}, y_{n}\right) \rightarrow f(x, y)$ as $n \rightarrow \infty$ if $\left(x_{n}, y_{n}\right) \rightarrow(x, y)$ as $n \rightarrow \infty$, since

$$
\lim _{n \rightarrow \infty} \log \left(1+\frac{x_{n}}{n}\right)^{n}=\log \left(\mathrm{e}^{x}\right)=x
$$

and $\lim _{n \rightarrow \infty} \int_{0}^{1}\left(1+\frac{x_{n}}{n}\right)^{s} \mathrm{~d} s=1$, if $x_{n} \rightarrow x$ as $n \rightarrow \infty$, since the function $\mathbb{R}_{++} \ni u \mapsto$ $\int_{0}^{1} u^{s} \mathrm{~d} s \in \mathbb{R}$ is continuous. Consequently, (3.9) implies (3.7).

Next we apply Lemma D.1 with $S=T=\mathbb{R}^{2}, C=\mathbb{R}^{2}$,

$$
\xi_{n}=\left[\begin{array}{c}
n^{3 / 2}\left(\widehat{\varrho}_{n}-\varrho\right) \\
n^{1 / 2}\left(\widehat{\bar{\beta}}_{n}-\bar{\beta}\right)
\end{array}\right], \quad \xi \stackrel{\mathcal{D}}{=} \mathcal{N}_{2}\left(\mathbf{0}, \int_{0}^{\infty} z^{2} \nu(\mathrm{d} z)\left[\begin{array}{cc}
\frac{1}{3}(\widetilde{\beta})^{2} & \frac{1}{2} \widetilde{\beta} \\
\frac{1}{2} \widetilde{\beta} & 1
\end{array}\right]\right)
$$


with functions $f: \mathbb{R}^{2} \rightarrow \mathbb{R}^{2}$ and $f_{n}: \mathbb{R}^{2} \rightarrow \mathbb{R}^{2}, n \in \mathbb{N}$, given by

$$
f\left(\left[\begin{array}{l}
x \\
y
\end{array}\right]\right):=\left[\begin{array}{l}
x \\
y
\end{array}\right], \quad(x, y) \in \mathbb{R}^{2}, \quad f_{n}\left(\left[\begin{array}{l}
x \\
y
\end{array}\right]\right):=\left[\begin{array}{c}
n^{3 / 2} \log \left(1+\frac{x}{n^{3 / 2}}\right) \\
n^{1 / 2}\left(\frac{n^{-1 / 2} y+\widetilde{\beta}}{\int_{0}^{1}\left(1+\frac{x}{n^{3 / 2}}\right)^{s} \mathrm{~d} s}-\widetilde{\beta}\right)
\end{array}\right]
$$

for $(x, y) \in \mathbb{R}^{2}$ with $x>-n^{3 / 2}$, and $f_{n}(x, y):=(0,0)$ otherwise. We have again $f_{n}\left(x_{n}, y_{n}\right) \rightarrow$ $f(x, y)$ as $n \rightarrow \infty$ if $\left(x_{n}, y_{n}\right) \rightarrow(x, y)$ as $n \rightarrow \infty$. Indeed,

$$
n^{1 / 2}\left(\frac{n^{-1 / 2} y_{n}+\widetilde{\beta}}{\int_{0}^{1}\left(1+\frac{x_{n}}{n^{3 / 2}}\right)^{s} \mathrm{~d} s}-\widetilde{\beta}\right)=\frac{y_{n}}{\int_{0}^{1}\left(1+\frac{x_{n}}{n^{3 / 2}}\right)^{s} \mathrm{~d} s}+\frac{\widetilde{\beta} n^{1 / 2}\left(1-\int_{0}^{1}\left(1+\frac{x_{n}}{n^{3 / 2}}\right)^{s} \mathrm{~d} s\right)}{\int_{0}^{1}\left(1+\frac{x_{n}}{n^{3 / 2}}\right)^{s} \mathrm{~d} s}
$$

if $x_{n}>-n^{3 / 2}$. Moreover,

$$
\begin{aligned}
& \left|n^{1 / 2}\left(1-\int_{0}^{1}\left(1+\frac{x_{n}}{n^{3 / 2}}\right)^{s} \mathrm{~d} s\right)-n^{1 / 2}\left(1-\int_{0}^{1}\left(1+\frac{x}{n^{3 / 2}}\right)^{s} \mathrm{~d} s\right)\right| \\
& =n^{1 / 2}\left|\frac{x_{n}-x}{n^{3 / 2}} \int_{0}^{1} s\left(1+\frac{\theta_{n}}{n^{3 / 2}}\right)^{s-1} \mathrm{~d} s\right| \leqslant K \frac{\left|x_{n}-x\right|}{n} \rightarrow 0 \quad \text { as } n \rightarrow \infty
\end{aligned}
$$

with $\theta_{n}$ (depending on $x_{n}$ and $x$ ) lying between $x_{n}$ and $x$, and with some appropriate $K>0$. Further, by L'Hospital's rule,

$$
\begin{aligned}
\lim _{n \rightarrow \infty} n^{1 / 2}\left(1-\int_{0}^{1}\left(1+\frac{x}{n^{3 / 2}}\right)^{s} \mathrm{~d} s\right) & =\lim _{h \rightarrow 0} \frac{1-\int_{0}^{1}\left(1+h^{3} x\right)^{s} \mathrm{~d} s}{h} \\
& =-\lim _{h \rightarrow 0} 3 h^{2} x \int_{0}^{1} s\left(1+h^{3} x\right)^{s-1} \mathrm{~d} s=0 .
\end{aligned}
$$

Consequently, (3.10) implies (3.8).

Theorem 3.4 will follow from the following statements by the continuous mapping theorem and by Slutsky's lemma, see below.

3.5 Theorem. Under the assumptions of Theorem 3.1, we have

$$
\sum_{k=1}^{n}\left[\begin{array}{c}
n^{-2} X_{k-1} \\
n^{-3} X_{k-1}^{2} \\
n^{-1} M_{k} \\
n^{-2} M_{k} X_{k-1}
\end{array}\right] \stackrel{\mathcal{D}}{\longrightarrow}\left[\begin{array}{c}
\int_{0}^{1} \mathcal{Y}_{t} \mathrm{~d} t \\
\int_{0}^{1} \mathcal{Y}_{t}^{2} \mathrm{~d} t \\
\mathcal{M}_{1} \\
\int_{0}^{1} \mathcal{Y}_{t} \mathrm{~d} \mathcal{M}_{t}
\end{array}\right] \quad \text { as } n \rightarrow \infty
$$

In case of $C=0$ the third and fourth coordinates of the limit vector is 0 in Theorem 3.5 , since $\left(\mathcal{Y}_{t}\right)_{t \in \mathbb{R}_{+}}$is the deterministic function $\mathcal{Y}_{t}=\widetilde{\beta} t, t \in \mathbb{R}_{+}$(see Remark 2.7), hence other scaling factors should be chosen for these coordinates, as given in the following theorem. 
3.6 Theorem. Suppose that the assumptions of Theorem 3.1 hold. If $C=0$, then

$$
\begin{gathered}
n^{-2} \sum_{k=1}^{n} X_{k-1} \stackrel{\mathbb{P}}{\longrightarrow} \frac{\widetilde{\beta}}{2} \quad \text { as } n \rightarrow \infty, \\
n^{-3} \sum_{k=1}^{n} X_{k-1}^{2} \stackrel{\mathbb{P}}{\longrightarrow} \frac{(\widetilde{\beta})^{2}}{3} \quad \text { as } n \rightarrow \infty, \\
\sum_{k=1}^{n}\left[\begin{array}{c}
n^{-1 / 2} M_{k} \\
n^{-3 / 2} M_{k} X_{k-1}
\end{array}\right] \stackrel{\mathcal{D}}{\longrightarrow} \mathcal{N}_{2}\left(\mathbf{0}, \int_{0}^{\infty} z^{2} \nu(\mathrm{d} z)\left[\begin{array}{cc}
1 & \frac{1}{2} \widetilde{\beta} \\
\frac{1}{2} \widetilde{\beta} & \frac{1}{3}(\widetilde{\beta})^{2}
\end{array}\right]\right) \quad \text { as } n \rightarrow \infty .
\end{gathered}
$$

Proof of Theorem 3.4. The statements about the existence of unique CLS estimators $\left(\widehat{\varrho}_{n}, \widehat{\bar{\beta}}_{n}\right)$ under the given conditions follow from Lemma C.1.

In order to derive (3.9) from Theorem [3.5, we can use the continuous mapping theorem. Indeed,

$$
\left[\begin{array}{c}
\widehat{\varrho}_{n}-\varrho \\
\widehat{\bar{\beta}}_{n}-\bar{\beta}
\end{array}\right]=\frac{1}{n \sum_{k=1}^{n} X_{k-1}^{2}-\left(\sum_{k=1}^{n} X_{k-1}\right)^{2}}\left[\begin{array}{c}
n \sum_{k=1}^{n} M_{k} X_{k-1}-\sum_{k=1}^{n} M_{k} \sum_{k=1}^{n} X_{k-1} \\
\sum_{k=1}^{n} M_{k} \sum_{k=1}^{n} X_{k-1}^{2}-\sum_{k=1}^{n} M_{k} X_{k-1} \sum_{k=1}^{n} X_{k-1}
\end{array}\right]
$$

on the set $H_{n}$. Moreover, since $\widetilde{\beta} \neq 0$, by the $\operatorname{SDE}(2.9)$, we have $\mathbb{P}\left(\mathcal{Y}_{t}=0, t \in\right.$ $[0,1])=0$, which implies $\mathbb{P}\left(\int_{0}^{1} \mathcal{Y}_{t}^{2} \mathrm{~d} t>0\right)=1$. By Remark 2.6. $\mathbb{P}\left(\mathcal{Y}_{t} \geqslant 0, t \in \mathbb{R}_{+}\right)=1$, and hence $\mathbb{P}\left(\int_{0}^{1} \mathcal{Y}_{t} \mathrm{~d} t>0\right)=1$. Next we show $\mathbb{P}\left(\int_{0}^{1} \mathcal{Y}_{t}^{2} \mathrm{~d} t-\left(\int_{0}^{1} \mathcal{Y}_{t} \mathrm{~d} t\right)^{2}>0\right)=1$. We have $\int_{0}^{1} \mathcal{Y}_{t}^{2} \mathrm{~d} t-\left(\int_{0}^{1} \mathcal{Y}_{t} \mathrm{~d} t\right)^{2}=\int_{0}^{1}\left(\mathcal{Y}_{t}-\int_{0}^{1} \mathcal{Y}_{s} \mathrm{~d} s\right)^{2} \mathrm{~d} t \geqslant 0$, and equality holds if and only if $\mathcal{Y}_{t}=\int_{0}^{1} \mathcal{Y}_{s} \mathrm{~d} s$ for almost every $t \in[0,1]$. Since $\mathcal{Y}$ has continuous sample paths almost surely, $\mathbb{P}\left(\int_{0}^{1} \mathcal{Y}_{t}^{2} \mathrm{~d} t-\left(\int_{0}^{1} \mathcal{Y}_{t} \mathrm{~d} t\right)^{2}=0\right)>0$ holds if and only if $\mathbb{P}\left(\mathcal{Y}_{t}=\int_{0}^{1} \mathcal{Y}_{s} \mathrm{~d} s, \forall t \in[0,1]\right)>0$. Hence, since $\mathcal{Y}_{0}=0$, this holds if and only if $P\left(\mathcal{Y}_{t}=0, \forall t \in[0,1]\right)>0$, which is a contradiction due to our assumption $\widetilde{\beta} \in \mathbb{R}_{++}$. Indeed, with the notations of the proof of Theorem 3.1 in Barczy et al. [1], $\left\{\omega \in \Omega: Y_{t}(\omega)=0, \forall t \in[0,1]\right\}=\widetilde{A}_{1} \cap A_{1}=\emptyset$. Consequently,

$$
\left[\begin{array}{c}
n\left(\widehat{\varrho}_{n}-\varrho\right) \\
\overline{\bar{\beta}}_{n}-\bar{\beta}
\end{array}\right] \stackrel{\mathcal{D}}{\longrightarrow} \frac{1}{\int_{0}^{1} \mathcal{Y}_{t}^{2} \mathrm{~d} t-\left(\int_{0}^{1} \mathcal{Y}_{t} \mathrm{~d} t\right)^{2}}\left[\begin{array}{c}
\int_{0}^{1} \mathcal{Y}_{t} \mathrm{~d} \mathcal{M}_{t}-\mathcal{M}_{1} \int_{0}^{1} \mathcal{Y}_{t} \mathrm{~d} t \\
\mathcal{M}_{1} \int_{0}^{1} \mathcal{Y}_{t}^{2} \mathrm{~d} t-\int_{0}^{1} \mathcal{Y}_{t} \mathrm{~d} t \int_{0}^{1} \mathcal{Y}_{t} \mathrm{~d} \mathcal{M}_{t}
\end{array}\right]
$$

as $n \rightarrow \infty$, and we obtain (3.9).

If, in addition, $c=0$ and $\mu=0$, then we derive (3.10) from Theorem 3.6 applying the continuous mapping theorem and Slutsky's lemma. We have

$$
\frac{1}{n^{3}} \sum_{k=1}^{n} X_{k-1}^{2}-\left(\frac{1}{n^{2}} \sum_{k=1}^{n} X_{k-1}\right)^{2} \stackrel{\mathbb{P}}{\longrightarrow} \frac{(\widetilde{\beta})^{2}}{3}-\left(\frac{\widetilde{\beta}}{2}\right)^{2}=\frac{(\widetilde{\beta})^{2}}{12} \quad \text { as } n \rightarrow \infty .
$$


Moreover,

$$
\begin{aligned}
& n^{-4}\left[\begin{array}{c}
n \sum_{k=1}^{n} M_{k} X_{k-1}-\sum_{k=1}^{n} M_{k} \sum_{k=1}^{n} X_{k-1} \\
\sum_{k=1}^{n} M_{k} \sum_{k=1}^{n} X_{k-1}^{2}-\sum_{k=1}^{n} M_{k} X_{k-1} \sum_{k=1}^{n} X_{k-1}
\end{array}\right] \\
& =n^{-4}\left[\begin{array}{cc}
-n^{1 / 2} \sum_{k=1}^{n} X_{k-1} & n^{5 / 2} \\
n^{1 / 2} \sum_{k=1}^{n} X_{k-1}^{2} & -n^{3 / 2} \sum_{k=1}^{n} X_{k-1}
\end{array}\right]\left[\begin{array}{c}
n^{-1 / 2} \sum_{k=1}^{n} M_{k} \\
n^{-3 / 2} \sum_{k=1}^{n} M_{k} X_{k-1}
\end{array}\right] \\
& =\left[\begin{array}{cc}
n^{-3 / 2} & 0 \\
0 & n^{-1 / 2}
\end{array}\right]\left[\begin{array}{cc}
-n^{-2} \sum_{k=1}^{n} X_{k-1} & 1 \\
n^{-3} \sum_{k=1}^{n} X_{k-1}^{2} & -n^{-2} \sum_{k=1}^{n} X_{k-1}
\end{array}\right]\left[\begin{array}{c}
n^{-1 / 2} \sum_{k=1}^{n} M_{k} \\
n^{-3 / 2} \sum_{k=1}^{n} M_{k} X_{k-1}
\end{array}\right] \text {, }
\end{aligned}
$$

hence, by Theorem 3.6 and Slutsky's lemma,

$$
\left[\begin{array}{c}
n^{3 / 2}\left(\widehat{\varrho}_{n}-\varrho\right) \\
n^{1 / 2}\left(\widehat{\bar{\beta}}_{n}-\bar{\beta}\right)
\end{array}\right]=\left[\begin{array}{cc}
n^{3 / 2} & 0 \\
0 & n^{1 / 2}
\end{array}\right]\left[\begin{array}{c}
\widehat{\varrho}_{n}-\varrho \\
\widehat{\bar{\beta}}_{n}-\bar{\beta}
\end{array}\right] \stackrel{\mathcal{D}}{\longrightarrow} \mathcal{N}_{2}(\mathbf{0}, \boldsymbol{\Sigma}),
$$

as $n \rightarrow \infty$, where

$$
\begin{aligned}
\boldsymbol{\Sigma} & :=\left(\frac{12}{(\widetilde{\beta})^{2}}\right)^{2} \int_{0}^{\infty} z^{2} \nu(\mathrm{d} z)\left[\begin{array}{cc}
-\frac{1}{2} \widetilde{\beta} & 1 \\
\frac{1}{3}(\widetilde{\beta})^{2} & -\frac{1}{2} \widetilde{\beta}
\end{array}\right]\left[\begin{array}{cc}
1 & \frac{1}{2} \widetilde{\beta} \\
\frac{1}{2} \widetilde{\beta} & \frac{1}{3}(\widetilde{\beta})^{2}
\end{array}\right]\left[\begin{array}{cc}
-\frac{1}{2} \widetilde{\beta} & \frac{1}{3}(\widetilde{\beta})^{2} \\
1 & -\frac{1}{2} \widetilde{\beta}
\end{array}\right] \\
& =\left(\frac{12}{(\widetilde{\beta})^{2}}\right)^{2} \int_{0}^{\infty} z^{2} \nu(\mathrm{d} z)\left[\begin{array}{cc}
\frac{1}{12}(\widetilde{\beta})^{2} & -\frac{1}{24}(\widetilde{\beta})^{3} \\
-\frac{1}{24}(\widetilde{\beta})^{3} & \frac{1}{36}(\widetilde{\beta})^{4}
\end{array}\right]=\frac{12}{(\widetilde{\beta})^{2}} \int_{0}^{\infty} z^{2} \nu(\mathrm{d} z)\left[\begin{array}{cc}
1 & -\frac{1}{2} \widetilde{\beta} \\
-\frac{1}{2} \widetilde{\beta} & \frac{1}{3}(\widetilde{\beta})^{2}
\end{array}\right],
\end{aligned}
$$

and we obtain (3.10).

\section{Proof of Theorem 3.5}

Consider the sequence of stochastic processes

$$
\mathcal{Z}_{t}^{(n)}:=\left[\begin{array}{c}
\mathcal{M}_{t}^{(n)} \\
\mathcal{N}_{t}^{(n)}
\end{array}\right]:=\sum_{k=1}^{\lfloor n t\rfloor} \boldsymbol{Z}_{k}^{(n)} \quad \text { with } \quad \boldsymbol{Z}_{k}^{(n)}:=\left[\begin{array}{c}
n^{-1} M_{k} \\
n^{-2} M_{k} X_{k-1}
\end{array}\right]
$$

for $t \in \mathbb{R}_{+}$and $k, n \in \mathbb{N}$. Theorem 3.5 follows from the following theorem (this will be explained after Theorem 4.1).

4.1 Theorem. Under the assumptions of Theorem 3.1, we have

$$
\mathcal{Z}^{(n)} \stackrel{\mathcal{D}}{\longrightarrow} \mathcal{Z}, \quad \text { as } n \rightarrow \infty,
$$

where the process $\left(\mathcal{Z}_{t}\right)_{t \in \mathbb{R}_{+}}$with values in $\mathbb{R}^{2}$ is the pathwise unique strong solution of the SDE

$$
\mathrm{d} \mathcal{Z}_{t}=\gamma\left(t, \mathcal{Z}_{t}\right) \mathrm{d} \mathcal{W}_{t}, \quad t \in \mathbb{R}_{+},
$$


with initial value $\mathcal{Z}_{0}=\mathbf{0}$, where $\left(\mathcal{W}_{t}\right)_{t \in \mathbb{R}_{+}}$is a standard Wiener process, and $\gamma: \mathbb{R}_{+} \times \mathbb{R}^{2} \rightarrow \mathbb{R}$ is defined by

$$
\gamma(t, \boldsymbol{x}):=\left[\begin{array}{l}
C^{1 / 2}\left(\left(x_{1}+\widetilde{\beta} t\right)^{+}\right)^{1 / 2} \\
C^{1 / 2}\left(\left(x_{1}+\widetilde{\beta} t\right)^{+}\right)^{3 / 2}
\end{array}\right], \quad t \in \mathbb{R}_{+}, \quad \boldsymbol{x}=\left(x_{1}, x_{2}\right)^{\top} \in \mathbb{R}^{2} .
$$

(Note that the statement of Theorem 4.1 holds even if $C=0$.)

The SDE (4.2) has the form

$$
\mathrm{d} \mathcal{Z}_{t}=:\left[\begin{array}{c}
\mathrm{d} \mathcal{M}_{t} \\
\mathrm{~d} \mathcal{N}_{t}
\end{array}\right]=\left[\begin{array}{l}
C^{1 / 2}\left(\left(\mathcal{M}_{t}+\widetilde{\beta} t\right)^{+}\right)^{1 / 2} \mathrm{~d} \mathcal{W}_{t} \\
C^{1 / 2}\left(\left(\mathcal{M}_{t}+\widetilde{\beta} t\right)^{+}\right)^{3 / 2} \mathrm{~d} \mathcal{W}_{t}
\end{array}\right], \quad t \in \mathbb{R}_{+} .
$$

One can prove that the first equation of the SDE (4.3) has a pathwise unique strong solution $\left(\mathcal{M}_{t}^{\left(y_{0}\right)}\right)_{t \in \mathbb{R}_{+}}$with arbitrary initial value $\mathcal{M}_{0}^{\left(y_{0}\right)}=y_{0} \in \mathbb{R}$. Indeed, it is equivalent to the existence of a pathwise unique strong solution of the SDE

$$
\mathrm{d} \mathcal{S}_{t}^{\left(y_{0}\right)}=\widetilde{\beta} \mathrm{d} t+C^{1 / 2}\left(\left(\mathcal{S}_{t}^{\left(y_{0}\right)}\right)^{+}\right)^{1 / 2} \mathrm{~d} \mathcal{W}_{t}, \quad t \in \mathbb{R}_{+},
$$

with initial value $\mathcal{S}_{0}^{\left(y_{0}\right)}=y_{0}$, since we have the correspondences

$$
\mathcal{S}_{t}^{\left(y_{0}\right)}=\mathcal{M}_{t}^{\left(y_{0}\right)}+\widetilde{\beta} t, \quad \mathcal{M}_{t}^{\left(y_{0}\right)}=\mathcal{S}_{t}^{\left(y_{0}\right)}-\widetilde{\beta} t
$$

by Itô's formula. By Remark 2.6, the SDE (4.4) has a pathwise unique strong solution $\left(\mathcal{S}_{t}^{\left(y_{0}\right)}\right)_{t \in \mathbb{R}_{+}}$for all initial values $\mathcal{S}_{0}^{\left(y_{0}\right)}=y_{0} \in \mathbb{R}$, and $\left(\mathcal{S}_{t}^{\left(y_{0}\right)}\right)^{+}$may be replaced by $\mathcal{S}_{t}^{\left(y_{0}\right)}$ for all $t \in \mathbb{R}_{+}$in (4.4) provided that $y_{0} \in \mathbb{R}_{+}$, hence $\left(\mathcal{M}_{t}+\widetilde{\beta} t\right)^{+}$may be replaced by $\mathcal{M}_{t}+\widetilde{\beta} t$ for all $t \in \mathbb{R}_{+}$in (4.3). Thus the SDE (4.2) has a pathwise unique strong solution with initial value $\mathcal{Z}_{0}=\mathbf{0}$, and we have

$$
\mathcal{Z}_{t}=\left[\begin{array}{c}
\mathcal{M}_{t} \\
\mathcal{N}_{t}
\end{array}\right]=\left[\begin{array}{c}
\int_{0}^{t} C^{1 / 2}\left(\mathcal{M}_{s}+\widetilde{\beta} s\right)^{1 / 2} \mathrm{~d} \mathcal{W}_{s} \\
\int_{0}^{t}\left(\mathcal{M}_{s}+\widetilde{\beta} s\right) \mathrm{d} \mathcal{M}_{s}
\end{array}\right], \quad t \in \mathbb{R}_{+}
$$

By continuous mapping theorem (see, e.g., the method of the proof of $\mathcal{X}^{(n)} \stackrel{\mathcal{D}}{\longrightarrow} \mathcal{X}$ in Theorem 3.1 in Barczy et al. [2]), one can easily derive

$$
\left[\begin{array}{l}
\mathcal{X}^{(n)} \\
\mathcal{Z}^{(n)}
\end{array}\right] \stackrel{\mathcal{D}}{\longrightarrow}\left[\begin{array}{l}
\tilde{\mathcal{X}} \\
\mathcal{Z}
\end{array}\right], \quad \text { as } n \rightarrow \infty
$$

where

$$
\mathcal{X}_{t}^{(n)}=n^{-1} X_{\lfloor n t\rfloor}, \quad \widetilde{\mathcal{X}}_{t}:=\mathcal{M}_{t}+\widetilde{\beta} t, \quad t \in \mathbb{R}_{+}, \quad n \in \mathbb{N} .
$$

By Itô's formula and the first equation of the SDE (4.3) we obtain

$$
\mathrm{d} \widetilde{\mathcal{X}}_{t}=\widetilde{\beta} \mathrm{d} t+C^{1 / 2}\left(\widetilde{\mathcal{X}}_{t}^{+}\right)^{1 / 2} \mathrm{~d} \mathcal{W}_{t}, \quad t \in \mathbb{R}_{+},
$$


hence the process $\left(\widetilde{\mathcal{X}}_{t}\right)_{t \in \mathbb{R}_{+}}$satisfies the $\operatorname{SDE}(\underline{2.9})$. Consequently, $\widetilde{\mathcal{X}}=\mathcal{Y}$. Next, by continuous mapping theorem, convergence (4.5) implies (3.11), see, e.g., the method of the proof of Proposition 3.1 in Barczy et al. [3].

Proof of Theorem 4.1. In order to show convergence $\mathcal{Z}^{(n)} \stackrel{\mathcal{D}}{\longrightarrow} \mathcal{Z}$, we apply Theorem E.1 with the special choices $\mathcal{U}:=\mathcal{Z}, \quad \boldsymbol{U}_{k}^{(n)}:=\boldsymbol{Z}_{k}^{(n)}, n, k \in \mathbb{N}, \quad\left(\mathcal{F}_{k}^{(n)}\right)_{k \in \mathbb{Z}_{+}}:=\left(\mathcal{F}_{k}\right)_{k \in \mathbb{Z}_{+}}$and the function $\gamma$ which is defined in Theorem 4.1. Note that the discussion after Theorem 4.1 shows that the SDE (4.2) admits a pathwise unique strong solution $\left(\mathcal{Z}_{t}^{\boldsymbol{z}}\right)_{t \in \mathbb{R}_{+}}$for all initial values $\mathcal{Z}_{0}^{\boldsymbol{z}}=\boldsymbol{z} \in \mathbb{R}^{2}$. Applying Cauchy-Schwarz inequality and Corollary B.5, one can check that $\mathbb{E}\left(\left\|\boldsymbol{U}_{k}^{(n)}\right\|^{2}\right)<\infty$ for all $n, k \in \mathbb{N}$.

Now we show that conditions (i) and (ii) of Theorem E.1 hold. The conditional variance has the form

$$
\operatorname{Var}\left(\boldsymbol{Z}_{k}^{(n)} \mid \mathcal{F}_{k-1}\right)=\operatorname{Var}\left(M_{k} \mid \mathcal{F}_{k-1}\right)\left[\begin{array}{cc}
n^{-2} & n^{-3} X_{k-1} \\
n^{-3} X_{k-1} & n^{-4} X_{k-1}^{2}
\end{array}\right]
$$

for $n \in \mathbb{N}, k \in\{1, \ldots, n\}$, and

$$
\gamma\left(s, \mathcal{Z}_{s}^{(n)}\right) \gamma\left(s, \mathcal{Z}_{s}^{(n)}\right)^{\top}=C\left[\begin{array}{cc}
\mathcal{M}_{s}^{(n)}+\widetilde{\beta} s & \left(\mathcal{M}_{s}^{(n)}+\widetilde{\beta} s\right)^{2} \\
\left(\mathcal{M}_{s}^{(n)}+\widetilde{\beta} s\right)^{2} & \left(\mathcal{M}_{s}^{(n)}+\widetilde{\beta} s\right)^{3}
\end{array}\right]
$$

for $s \in \mathbb{R}_{+}$, where we used that $\left(\mathcal{M}_{s}^{(n)}+\widetilde{\beta} s\right)^{+}=\mathcal{M}_{s}^{(n)}+\widetilde{\beta} s, \quad s \in \mathbb{R}_{+}, \quad n \in \mathbb{N}$. Indeed, by (3.3), we get

$$
\mathcal{M}_{s}^{(n)}+\widetilde{\beta} s=\frac{1}{n} \sum_{k=1}^{\lfloor n s\rfloor}\left(X_{k}-\mathrm{e}^{\widetilde{b}} X_{k-1}-\bar{\beta}\right)+\widetilde{\beta} s=\frac{1}{n} X_{\lfloor n s\rfloor}+\frac{n s-\lfloor n s\rfloor}{n} \widetilde{\beta} \in \mathbb{R}_{+}
$$

for $s \in \mathbb{R}_{+}, n \in \mathbb{N}$, since $\mathrm{e}^{\widetilde{b}}=1$ and $\bar{\beta}=\widetilde{\beta}$.

In order to check condition (i) of Theorem E.1, we need to prove that for each $T>0$, as $n \rightarrow \infty$,

$$
\begin{gathered}
\sup _{t \in[0, T]}\left|\frac{1}{n^{2}} \sum_{k=1}^{\lfloor n t\rfloor} \operatorname{Var}\left(M_{k} \mid \mathcal{F}_{k-1}\right)-C \int_{0}^{t}\left(\mathcal{M}_{s}^{(n)}+\widetilde{\beta} s\right) \mathrm{d} s\right| \stackrel{\mathbb{P}}{\longrightarrow} 0, \\
\sup _{t \in[0, T]}\left|\frac{1}{n^{3}} \sum_{k=1}^{\lfloor n t\rfloor} X_{k-1} \operatorname{Var}\left(M_{k} \mid \mathcal{F}_{k-1}\right)-C \int_{0}^{t}\left(\mathcal{M}_{s}^{(n)}+\widetilde{\beta} s\right)^{2} \mathrm{~d} s\right| \stackrel{\mathbb{P}}{\longrightarrow} 0, \\
\sup _{t \in[0, T]}\left|\frac{1}{n^{4}} \sum_{k=1}^{\lfloor n t\rfloor} X_{k-1}^{2} \operatorname{Var}\left(M_{k} \mid \mathcal{F}_{k-1}\right)-C \int_{0}^{t}\left(\mathcal{M}_{s}^{(n)}+\widetilde{\beta} s\right)^{3} \mathrm{~d} s\right| \stackrel{\mathbb{P}}{\longrightarrow} 0 .
\end{gathered}
$$

First we show (4.7). By (4.6), $\int_{0}^{t}\left(\mathcal{M}_{s}^{(n)}+s \widetilde{\beta}\right) \mathrm{d} s$ has the form

$$
\frac{1}{n^{2}} \sum_{k=1}^{\lfloor n t\rfloor-1} X_{k}+\frac{n t-\lfloor n t\rfloor}{n^{2}} X_{\lfloor n t\rfloor}+\frac{\lfloor n t\rfloor+(n t-\lfloor n t\rfloor)^{2}}{2 n^{2}} \widetilde{\beta} .
$$


By Proposition B.3 and $\widetilde{b}=0$,

$$
\operatorname{Var}\left(M_{k} \mid \mathcal{F}_{k-1}\right)=V X_{k-1}+V_{0}=C X_{k-1}+V_{0} .
$$

Thus, in order to show (4.7), it suffices to prove

$$
\begin{gathered}
n^{-2} \sup _{t \in[0, T]} X_{\lfloor n t\rfloor} \stackrel{\mathbb{P}}{\longrightarrow} 0, \\
n^{-2} \sup _{t \in[0, T]}\left[\lfloor n t\rfloor+(n t-\lfloor n t\rfloor)^{2}\right] \rightarrow 0,
\end{gathered}
$$

as $n \rightarrow \infty$. Using (B.5) with $(\ell, i)=(2,1)$, we have (4.11). Clearly, (4.12) follows from $|n t-\lfloor n t\rfloor| \leqslant 1, \quad n \in \mathbb{N}, \quad t \in \mathbb{R}_{+}$, thus we conclude (4.7).

Next we turn to prove (4.8). By (4.6),

$$
\begin{aligned}
\int_{0}^{t}\left(\mathcal{M}_{s}^{(n)}+s \widetilde{\beta}\right)^{2} \mathrm{~d} s= & \frac{1}{n^{3}} \sum_{k=1}^{\lfloor n t\rfloor-1} X_{k}^{2}+\frac{1}{n^{3}} \widetilde{\beta} \sum_{k=1}^{\lfloor n t\rfloor-1} X_{k}+\frac{n t-\lfloor n t\rfloor}{n^{3}} X_{\lfloor n t\rfloor}^{2} \\
& +\frac{(n t-\lfloor n t\rfloor)^{2}}{n^{3}} \widetilde{\beta} X_{\lfloor n t\rfloor}+\frac{\lfloor n t\rfloor+(n t-\lfloor n t\rfloor)^{3}}{3 n^{3}}(\widetilde{\beta})^{2} .
\end{aligned}
$$

Recalling formula (4.10), we obtain

$$
\sum_{k=1}^{\lfloor n t\rfloor} X_{k-1} \operatorname{Var}\left(M_{k} \mid \mathcal{F}_{k-1}\right)=C \sum_{k=1}^{\lfloor n t\rfloor} X_{k-1}^{2}+V_{0} \sum_{k=1}^{\lfloor n t\rfloor} X_{k-1} .
$$

Thus, in order to show (4.8), it suffices to prove

$$
\begin{gathered}
n^{-3} \sum_{k=1}^{\lfloor n T\rfloor} X_{k} \stackrel{\mathbb{P}}{\longrightarrow} 0, \\
n^{-3 / 2} \sup _{t \in[0, T]} X_{\lfloor n t\rfloor} \stackrel{\mathbb{P}}{\longrightarrow} 0, \\
n^{-3} \sup _{t \in[0, T]}\left[\lfloor n t\rfloor+(n t-\lfloor n t\rfloor)^{3}\right] \rightarrow 0
\end{gathered}
$$

as $n \rightarrow \infty$. Using (B.4) with $(\ell, i)=(2,1)$, we have (4.14). By (B.5) with $(\ell, i)=(3,1)$, we have (4.15). Clearly, (4.16) follows from $|n t-\lfloor n t\rfloor| \leqslant 1, n \in \mathbb{N}, t \in \mathbb{R}_{+}$, thus we conclude (4.8).

Now we turn to check (4.9). Again by (4.6), we have

$$
\begin{aligned}
\int_{0}^{t}\left(\mathcal{M}_{s}^{(n)}+s \widetilde{\beta}\right)^{3} \mathrm{~d} s= & \frac{1}{n^{4}} \sum_{k=1}^{\lfloor n t\rfloor-1} X_{k}^{3}+\frac{3}{2 n^{4}} \widetilde{\beta} \sum_{k=1}^{\lfloor n t\rfloor-1} X_{k}^{2}+\frac{1}{n^{4}}(\widetilde{\beta})^{2} \sum_{k=1}^{\lfloor n t\rfloor-1} X_{k} \\
& +\frac{n t-\lfloor n t\rfloor}{n^{4}} X_{\lfloor n t\rfloor}^{3}+\frac{3(n t-\lfloor n t\rfloor)^{2}}{2 n^{4}} \widetilde{\beta} X_{\lfloor n t\rfloor}^{2} \\
& +\frac{(n t-\lfloor n t\rfloor)^{3}}{n^{4}}(\widetilde{\beta})^{2} X_{\lfloor n t\rfloor}+\frac{\lfloor n t\rfloor+(n t-\lfloor n t\rfloor)^{4}}{4 n^{4}}(\widetilde{\beta})^{3} .
\end{aligned}
$$


Recalling formula (4.10), we obtain

$$
\sum_{k=1}^{\lfloor n t\rfloor} X_{k-1}^{2} \operatorname{Var}\left(M_{k} \mid \mathcal{F}_{k-1}\right)=C \sum_{k=1}^{\lfloor n t\rfloor} X_{k-1}^{3}+V_{0} \sum_{k=1}^{\lfloor n t\rfloor} X_{k-1}^{2} .
$$

Thus, in order to show (4.9), it suffices to prove

$$
\begin{gathered}
n^{-4} \sum_{k=1}^{\lfloor n T\rfloor} X_{k}^{2} \stackrel{\mathbb{P}}{\longrightarrow} 0, \\
n^{-4} \sum_{k=1}^{\lfloor n T\rfloor} X_{k} \stackrel{\mathbb{P}}{\longrightarrow} 0, \\
n^{-4 / 3} \sup _{t \in[0, T]} X_{\lfloor n t\rfloor} \stackrel{\mathbb{P}}{\longrightarrow} 0, \\
n^{-4} \sup _{t \in[0, T]}\left[\lfloor n t\rfloor+(n t-\lfloor n t\rfloor)^{4}\right] \rightarrow 0
\end{gathered}
$$

as $n \rightarrow \infty$. Using (B.4) with $(\ell, i)=(4,2)$ and $(\ell, i)=(2,1)$, we have (4.18) and (4.19), respectively. By (B.5) with $(\ell, i)=(4,1)$, we have (4.20). Clearly, (4.21) follows again from $|n t-\lfloor n t\rfloor| \leqslant 1, \quad n \in \mathbb{N}, \quad t \in \mathbb{R}_{+}$, thus we conclude (4.9). Note that the proof of (4.7)-(4.9) is essentially the same as the proof of (5.5)-(5.7) in Ispány et al. [13].

Finally, we check condition (ii) of Theorem E.1, that is, the conditional Lindeberg condition

$$
\sum_{k=1}^{\lfloor n T\rfloor} \mathbb{E}\left(\left\|\boldsymbol{Z}_{k}^{(n)}\right\|^{2} \mathbb{1}_{\left\{\left\|\boldsymbol{Z}_{k}^{(n)}\right\|>\theta\right\}} \mid \mathcal{F}_{k-1}\right) \stackrel{\mathbb{P}}{\longrightarrow} 0, \quad \text { as } n \rightarrow \infty
$$

for all $\theta>0$ and $T>0$. We have $\mathbb{E}\left(\left\|\boldsymbol{Z}_{k}^{(n)}\right\|^{2} \mathbb{1}_{\left\{\left\|\boldsymbol{Z}_{k}^{(n)}\right\|>\theta\right\}} \mid \mathcal{F}_{k-1}\right) \leqslant \theta^{-2} \mathbb{E}\left(\left\|\boldsymbol{Z}_{k}^{(n)}\right\|^{4} \mid \mathcal{F}_{k-1}\right)$ and

$$
\left\|\boldsymbol{Z}_{k}^{(n)}\right\|^{4} \leqslant 2\left(n^{-4}+n^{-8} X_{k-1}^{4}\right) M_{k}^{4} .
$$

Hence, for all $\theta>0$ and $T>0$, we have

$$
\sum_{k=1}^{\lfloor n T\rfloor} \mathbb{E}\left(\left\|\boldsymbol{Z}_{k}^{(n)}\right\|^{2} \mathbb{1}_{\left\{\left\|\boldsymbol{Z}_{k}^{(n)}\right\|>\theta\right\}}\right) \rightarrow 0, \quad \text { as } n \rightarrow \infty,
$$

since $\mathbb{E}\left(M_{k}^{4}\right)=\mathrm{O}\left(k^{2}\right)$ and $\mathbb{E}\left(M_{k}^{4} X_{k-1}^{4}\right) \leqslant \sqrt{\mathbb{E}\left(M_{k}^{8}\right) \mathbb{E}\left(X_{k-1}^{8}\right)}=\mathrm{O}\left(k^{6}\right)$ by Corollary B.5, This yields (4.22).

We call the attention that our moment conditions (2.7) with $q=8$ are used for applying Corollaries B.5 and B.6.

\section{$5 \quad$ Proof of Theorem 3.6}

The first two convergences in Theorem 3.6 follows from the following approximations. 
5.1 Lemma. Suppose that the assumptions of Theorem 3.1 hold. If $C=0$, then for each $T>0$,

$$
\sup _{t \in[0, T]}\left|\frac{1}{n^{2}} \sum_{k=1}^{\lfloor n t\rfloor} X_{k-1}-\widetilde{\beta} \frac{t^{2}}{2}\right| \stackrel{\mathbb{P}}{\longrightarrow} 0, \quad \text { as } n \rightarrow \infty
$$

Proof. We have

$$
\left|\frac{1}{n^{2}} \sum_{k=1}^{\lfloor n t\rfloor} X_{k-1}-\widetilde{\beta} \frac{t^{2}}{2}\right| \leqslant \frac{1}{n^{2}} \sum_{k=1}^{\lfloor n t\rfloor}\left|X_{k-1}-\widetilde{\beta}(k-1)\right|+\widetilde{\beta}\left|\frac{1}{n^{2}} \sum_{k=1}^{\lfloor n t\rfloor}(k-1)-\frac{t^{2}}{2}\right|,
$$

where

$$
\sup _{t \in[0, T]}\left|\frac{1}{n^{2}} \sum_{k=1}^{\lfloor n t\rfloor}(k-1)-\frac{t^{2}}{2}\right| \rightarrow 0, \quad \text { as } n \rightarrow \infty,
$$

hence, in order to show (5.1), it suffices to prove

$$
\frac{1}{n^{2}} \sum_{k=1}^{\lfloor n T\rfloor}\left|X_{k}-\widetilde{\beta} k\right| \stackrel{\mathbb{P}}{\longrightarrow} 0, \quad \text { as } n \rightarrow \infty .
$$

Recursion (3.4) yields $\mathbb{E}\left(X_{k}\right)=\mathbb{E}\left(X_{k-1}\right)+\widetilde{\beta}, \quad k \in \mathbb{N}$, with intital value $\mathbb{E}\left(X_{0}\right)=0$, hence $\mathbb{E}\left(X_{k}\right)=\widetilde{\beta} k, k \in \mathbb{N}$. For the sequence

$$
\widetilde{X}_{k}:=X_{k}-\mathbb{E}\left(X_{k}\right)=X_{k}-\widetilde{\beta} k, \quad k \in \mathbb{N},
$$

by (3.4), we get a recursion $\widetilde{X}_{k}=\widetilde{X}_{k-1}+M_{k}, k \in \mathbb{N}$, with intital value $\widetilde{X}_{0}=0$. Applying Doob's maximal inequality (see, e.g., Revuz and Yor [20, Chapter II, Theorem 1.7]) for the martingale $\widetilde{X}_{n}=\sum_{k=1}^{n} M_{k}, n \in \mathbb{N}$,

$$
\mathbb{E}\left(\sup _{t \in[0, T]}\left|\sum_{k=1}^{\lfloor n t\rfloor} M_{k}\right|^{2}\right) \leqslant 4 \mathbb{E}\left(\left|\sum_{k=1}^{\lfloor n T\rfloor} M_{k}\right|^{2}\right)=4 \sum_{k=1}^{\lfloor n T\rfloor} \mathbb{E}\left(M_{k}^{2}\right)=\mathrm{O}(n),
$$

where we applied Corollary B.5. Consequently,

$$
n^{-1} \max _{k \in\{1, \ldots,\lfloor n T\}\}}\left|X_{k}-\widetilde{\beta} k\right|=n^{-1} \max _{k \in\{1, \ldots,\lfloor n T\}\}}\left|\widetilde{X}_{k}\right| \stackrel{\mathbb{P}}{\longrightarrow} 0 \quad \text { as } n \rightarrow \infty .
$$

Thus,

$$
\frac{1}{n^{2}} \sum_{k=1}^{\lfloor n T\rfloor}\left|X_{k}-k \widetilde{\beta}\right| \leqslant \frac{\lfloor n T\rfloor}{n^{2}} \max _{k \in\{1, \ldots,\lfloor n T\rfloor\}}\left|X_{k}-k \widetilde{\beta}\right| \stackrel{\mathbb{P}}{\longrightarrow} 0,
$$

as $n \rightarrow \infty$, thus we conclude (5.2), and hence (5.1).

5.2 Lemma. Suppose that the assumptions of Theorem 3.1 hold. If $C=0$, then for each $T>0$,

$$
\sup _{t \in[0, T]}\left|\frac{1}{n^{3}} \sum_{k=1}^{\lfloor n t\rfloor} X_{k-1}^{2}-(\widetilde{\beta})^{2} \frac{t^{3}}{3}\right| \stackrel{\mathbb{P}}{\longrightarrow} 0, \quad \text { as } n \rightarrow \infty .
$$


Proof. We have

$$
\left|\frac{1}{n^{3}} \sum_{k=1}^{\lfloor n t\rfloor} X_{k-1}^{2}-(\widetilde{\beta})^{2} \frac{t^{3}}{3}\right| \leqslant \frac{1}{n^{3}} \sum_{k=1}^{\lfloor n t\rfloor}\left|X_{k-1}^{2}-(\widetilde{\beta})^{2}(k-1)^{2}\right|+(\widetilde{\beta})^{2}\left|\frac{1}{n^{3}} \sum_{k=1}^{\lfloor n t\rfloor}(k-1)^{2}-\frac{t^{3}}{3}\right|,
$$

where

$$
\sup _{t \in[0, T]}\left|\frac{1}{n^{3}} \sum_{k=1}^{\lfloor n t\rfloor}(k-1)^{2}-\frac{t^{3}}{3}\right| \rightarrow 0, \quad \text { as } n \rightarrow \infty,
$$

hence, in order to show (5.5), it suffices to prove

$$
\frac{1}{n^{3}} \sum_{k=1}^{\lfloor n T\rfloor}\left|X_{k}^{2}-(\widetilde{\beta})^{2} k^{2}\right| \stackrel{\mathbb{P}}{\longrightarrow} 0, \quad \text { as } n \rightarrow \infty .
$$

We have

$$
\left|X_{k}^{2}-k^{2}(\widetilde{\beta})^{2}\right| \leqslant\left|X_{k}-k \widetilde{\beta}\right|^{2}+2 k \widetilde{\beta}\left|X_{k}-k \widetilde{\beta}\right|,
$$

hence, by (5.4),

$$
\begin{aligned}
n^{-2} \max _{k \in\{1, \ldots,\lfloor n T\rfloor\}} \mid X_{k}^{2}- & k^{2}(\widetilde{\beta})^{2} \mid \\
& \leqslant\left(n^{-1} \max _{k \in\{1, \ldots,\lfloor n T\rfloor\}}\left|X_{k}-k \widetilde{\beta}\right|\right)^{2}+\frac{2\lfloor n T\rfloor}{n^{2}} \widetilde{\beta} \max _{k \in\{1, \ldots,\lfloor n T\rfloor\}}\left|X_{k}-k \widetilde{\beta}\right| \stackrel{\mathbb{P}}{\longrightarrow} 0,
\end{aligned}
$$

as $n \rightarrow \infty$. Thus,

$$
\frac{1}{n^{3}} \sum_{k=1}^{\lfloor n T\rfloor}\left|X_{k}^{2}-k^{2}(\widetilde{\beta})^{2}\right| \leqslant \frac{\lfloor n T\rfloor}{n^{3}} \max _{k \in\{1, \ldots,\lfloor n T\rfloor\}}\left|X_{k}^{2}-k^{2}(\widetilde{\beta})^{2}\right| \stackrel{\mathbb{P}}{\longrightarrow} 0,
$$

as $n \rightarrow \infty$, and we conclude (5.6), and hence (5.5).

The proof of the third convergence in Theorem 3.6 is similar to the proof of Theorem 3.5 . Consider the sequence of stochastic processes

$$
\mathcal{Z}_{t}^{(n)}:=\sum_{k=1}^{\lfloor n t\rfloor} \boldsymbol{Z}_{k}^{(n)} \quad \text { with } \quad \boldsymbol{Z}_{k}^{(n)}:=\left[\begin{array}{c}
n^{-1 / 2} M_{k} \\
n^{-3 / 2} M_{k} X_{k-1}
\end{array}\right]
$$

for $t \in \mathbb{R}_{+}$and $k, n \in \mathbb{N}$. The proof of the third convergence in Theorem 3.6 follows from Lemmas 5.1 and 5.2, and the following theorem.

5.3 Theorem. If $C=0$ then

$$
\mathcal{Z}^{(n)} \stackrel{\mathcal{D}}{\longrightarrow} \mathcal{Z}, \quad \text { as } n \rightarrow \infty,
$$

where the process $\left(\mathcal{Z}_{t}\right)_{t \in \mathbb{R}_{+}}$with values in $\mathbb{R}^{2}$ is the pathwise unique strong solution of the $S D E$

$$
\mathrm{d} \mathcal{Z}_{t}=\gamma(t) \mathrm{d} \widetilde{\mathcal{W}}_{t}, \quad t \in \mathbb{R}_{+},
$$


with initial value $\mathcal{Z}_{0}=\mathbf{0}$, where $\left(\widetilde{\mathcal{W}}_{t}\right)_{t \in \mathbb{R}_{+}}$is a 2-dimensional standard Wiener process, and $\gamma: \mathbb{R}_{+} \rightarrow \mathbb{R}^{2 \times 2}$ is defined by

$$
\gamma(t):=V_{0}\left[\begin{array}{cc}
1 & \widetilde{\beta} t \\
\widetilde{\beta} t & (\widetilde{\beta})^{2} t^{2}
\end{array}\right]^{1 / 2}, \quad t \in \mathbb{R}_{+},
$$

where $V_{0}=\int_{0}^{\infty} z^{2} \nu(\mathrm{d} z)$.

The SDE (5.8) has a pathwise unique strong solution with initial value $\mathcal{Z}_{0}=\mathbf{0}$, for which we have

$$
\mathcal{Z}_{t}=V_{0}^{1 / 2} \int_{0}^{t}\left[\begin{array}{cc}
1 & \widetilde{\beta} s \\
\widetilde{\beta} s & (\widetilde{\beta})^{2} s^{2}
\end{array}\right]^{1 / 2} \mathrm{~d} \widetilde{\mathcal{W}}_{s}, \quad t \in \mathbb{R}_{+} .
$$

Proof of Theorem 5.3. We follow again the method of the proof of Theorem 4.1. The conditional variance has the form

$$
\operatorname{Var}\left(\boldsymbol{Z}_{k}^{(n)} \mid \mathcal{F}_{k-1}\right)=\operatorname{Var}\left(M_{k} \mid \mathcal{F}_{k-1}\right)\left[\begin{array}{cc}
n^{-1} & n^{-2} X_{k-1} \\
n^{-2} X_{k-1} & n^{-3} X_{k-1}^{2}
\end{array}\right]
$$

for $n \in \mathbb{N}, k \in\{1, \ldots, n\}$. Moreover, $\gamma(s) \gamma(s)^{\top}$ takes the form

$$
\gamma(s) \gamma(s)^{\top}=V_{0}\left[\begin{array}{cc}
1 & \widetilde{\beta} s \\
\widetilde{\beta} s & (\widetilde{\beta})^{2} s^{2}
\end{array}\right], \quad s \in \mathbb{R}_{+} .
$$

In order to check condition (i) of Theorem E.1, we need to prove only that for each $T>0$,

$$
\begin{gathered}
\sup _{t \in[0, T]}\left|\frac{1}{n} \sum_{k=1}^{\lfloor n t\rfloor} \operatorname{Var}\left(M_{k} \mid \mathcal{F}_{k-1}\right)-V_{0} \int_{0}^{t} \mathrm{~d} s\right| \stackrel{\mathbb{P}}{\longrightarrow} 0, \\
\sup _{t \in[0, T]}\left|\frac{1}{n^{2}} \sum_{k=1}^{\lfloor n t\rfloor} X_{k-1} \operatorname{Var}\left(M_{k} \mid \mathcal{F}_{k-1}\right)-V_{0} \widetilde{\beta} \int_{0}^{t} s \mathrm{~d} s\right| \stackrel{\mathbb{P}}{\longrightarrow} 0, \\
\sup _{t \in[0, T]}\left|\frac{1}{n^{3}} \sum_{k=1}^{\lfloor n t\rfloor} X_{k-1}^{2} \operatorname{Var}\left(M_{k} \mid \mathcal{F}_{k-1}\right)-V_{0} \widetilde{\beta}^{2} \int_{0}^{t} s^{2} \mathrm{~d} s\right| \stackrel{\mathbb{P}}{\longrightarrow} 0,
\end{gathered}
$$

as $n \rightarrow \infty$.

By Proposition B.3, the assumption $C=0$ yields $\operatorname{Var}\left(M_{k} \mid \mathcal{F}_{k-1}\right)=V_{0}=\int_{0}^{\infty} z^{2} \nu(\mathrm{d} z)$, hence (5.9), (5.10) and (5.11) follow from Lemmas 5.1 and 5.2, respectively.

Finally, we check condition (ii) of Theorem E.1, that is, the conditional Lindeberg condition

$$
\sum_{k=1}^{\lfloor n T\rfloor} \mathbb{E}\left(\left\|\boldsymbol{Z}_{k}^{(n)}\right\|^{2} \mathbb{1}_{\left\{\left\|\boldsymbol{Z}_{k}^{(n)}\right\|>\theta\right\}} \mid \mathcal{F}_{k-1}\right) \stackrel{\mathbb{P}}{\longrightarrow} 0, \quad \text { as } n \rightarrow \infty
$$


for all $\theta>0$ and $T>0$. We have $\mathbb{E}\left(\left\|\boldsymbol{Z}_{k}^{(n)}\right\|^{2} \mathbb{1}_{\left\{\left\|\boldsymbol{Z}_{k}^{(n)}\right\|>\theta\right\}} \mid \mathcal{F}_{k-1}\right) \leqslant \theta^{-2} \mathbb{E}\left(\left\|\boldsymbol{Z}_{k}^{(n)}\right\|^{4} \mid \mathcal{F}_{k-1}\right)$ and

$$
\left\|\boldsymbol{Z}_{k}^{(n)}\right\|^{4} \leqslant 2\left(n^{-2}+n^{-6} X_{k-1}^{4}\right) M_{k}^{4} .
$$

Hence, for all $\theta>0$ and $T>0$, we have

$$
\sum_{k=1}^{\lfloor n T\rfloor} \mathbb{E}\left(\left\|\boldsymbol{Z}_{k}^{(n)}\right\|^{2} \mathbb{1}_{\left\{\left\|\boldsymbol{Z}_{k}^{(n)}\right\|>\theta\right\}}\right) \rightarrow 0, \quad \text { as } n \rightarrow \infty,
$$

since $\mathbb{E}\left(M_{k}^{4}\right)=\mathrm{O}(1)$ and $\mathbb{E}\left(M_{k}^{4} X_{k-1}^{4}\right) \leqslant \sqrt{\mathbb{E}\left(M_{k}^{8}\right) \mathbb{E}\left(X_{k-1}^{8}\right)}=\mathrm{O}\left(k^{4}\right)$ by Corollary B.5. This yields (5.12).

\section{Appendices}

\section{A SDE for CBI processes}

One can rewrite the SDE (1.1) in a form which does not contain integrals with respect to noncompensated Poisson random measures (see, SDE (2.6)), and then one can perform a linear transformation in order to remove randomness from the drift as follows, see Lemma 4.1 in Barczy et al. [6]. This form is very useful for handling $M_{k}, k \in \mathbb{N}$.

A.1 Lemma. Let $(c, \beta, b, \nu, \mu)$ be a set of admissible parameters such that the moment condition (2.3) holds. Let $\left(X_{t}\right)_{t \in \mathbb{R}_{+}}$be a pathwise unique $\mathbb{R}_{+}$-valued strong solution to the SDE (1.1) such that $\mathbb{E}\left(X_{0}\right)<\infty$. Then

$$
\begin{aligned}
X_{t}= & \mathrm{e}^{\widetilde{b}(t-s)} X_{s}+\int_{s}^{t} \mathrm{e}^{\widetilde{b}(t-u)} \widetilde{\beta} \mathrm{d} u+\int_{s}^{t} \mathrm{e}^{\widetilde{b}(t-u)} \sqrt{2 c X_{u}} \mathrm{~d} W_{u} \\
& +\int_{s}^{t} \int_{0}^{\infty} \int_{0}^{\infty} \mathrm{e}^{\widetilde{b}(t-u)} z \mathbb{1}_{\left\{v \leqslant X_{s-}\right\}} \widetilde{N}(\mathrm{~d} u, \mathrm{~d} z, \mathrm{~d} v)+\int_{s}^{t} \int_{0}^{\infty} \mathrm{e}^{\widetilde{b}(t-u)} z \widetilde{M}(\mathrm{~d} u, \mathrm{~d} z)
\end{aligned}
$$

for all $s, t \in \mathbb{R}_{+}$, with $s \leqslant t$. Consequently,

$$
\begin{aligned}
M_{k}= & \int_{k-1}^{k} \mathrm{e}^{\widetilde{b}(k-u)} \sqrt{2 c X_{u}} \mathrm{~d} W_{u}+\int_{k-1}^{k} \int_{0}^{\infty} \int_{0}^{\infty} \mathrm{e}^{\widetilde{b}(k-u)} z \mathbb{1}_{\left\{v \leqslant X_{s-}\right\}} \widetilde{N}(\mathrm{~d} u, \mathrm{~d} z, \mathrm{~d} v) \\
& +\int_{k-1}^{k} \int_{0}^{\infty} \mathrm{e}^{\widetilde{b}(k-u)} z \widetilde{M}(\mathrm{~d} u, \mathrm{~d} z), \quad k \in \mathbb{N} .
\end{aligned}
$$

Proof. The last statement follows from (3.3), since $\widetilde{\beta} \int_{k-1}^{k} \mathrm{e}^{\widetilde{b}(k-u)} \mathrm{d} u=\widetilde{\beta} \int_{0}^{1} \mathrm{e}^{\widetilde{b}(1-u)} \mathrm{d} u=\bar{\beta}$.

Note that the formulas for $\left(X_{t}\right)_{t \in \mathbb{R}_{+}}$and $\left(M_{k}\right)_{k \in \mathbb{N}}$ in Lemma A.1 can be found as the first displayed formula in the proof of Lemma 2.1 in Huang et al. [10, and formulas (1.5) and (1.7) in Li and Ma [18, respectively. 
A.2 Lemma. Let $\left(X_{t}\right)_{t \in \mathbb{R}_{+}}$be a CBI process with parameters $(c, \beta, b, \nu, \mu)$ such that $X_{0}=0$, $\beta \neq 0$ or $\nu \neq 0$, and $\widetilde{b}=0$ (hence it is critical). Suppose that $C=0$ and the moment conditions (2.7) hold with $q=2$. Then

$$
M_{k}=\int_{k-1}^{k} \int_{0}^{\infty} z \widetilde{M}(\mathrm{~d} u, \mathrm{~d} z), \quad k \in \mathbb{N} .
$$

and the sequence $\left(M_{k}\right)_{k \in \mathbb{N}}$ consists of independent and identically distributed random vectors.

Proof. The assumption $C=0$ implies $c=0$ and $\mu=0$ (see, Remark 2.7), thus, by Lemma A.1, we obtain the formula for $M_{k}, k \in \mathbb{N}$.

A Poisson point process admits independent increments, hence $M_{k}, k \in \mathbb{N}$, are independent.

For each $k \in \mathbb{N}$, the Laplace transform of the random variable $M_{k}$ has the form

$$
\begin{aligned}
\mathbb{E}\left(\mathrm{e}^{-\theta M_{k}}\right) & =\exp \left\{-\int_{k-1}^{k} \int_{0}^{\infty}\left(1-\mathrm{e}^{-\theta r}\right) \mathrm{d} s \nu(\mathrm{d} r)\right\} \\
& =\exp \left\{-\int_{0}^{1} \int_{0}^{\infty}\left(1-\mathrm{e}^{-\theta r}\right) \mathrm{d} u \nu(\mathrm{d} r)\right\}=\mathbb{E}\left(\mathrm{e}^{-\theta M_{1}}\right)
\end{aligned}
$$

for all $\theta \in \mathbb{R}_{+}$, see, i.e., Kyprianou [16, page 44], hence $M_{k}, k \in \mathbb{N}$, are identically distributed.

\section{B On moments of CBI processes}

In the proof of Theorem 3.1, good bounds for moments of the random variables $\left(M_{k}\right)_{k \in \mathbb{Z}_{+}}$ and $\left(X_{k}\right)_{k \in \mathbb{Z}_{+}}$are extensively used. The following estimates are proved in Barczy and Pap [7, Lemmas B.2 and B.3].

B.1 Lemma. Let $\left(X_{t}\right)_{t \in \mathbb{R}_{+}}$be a CBI process with parameters $(c, \beta, b, \nu, \mu)$ such that $\mathbb{E}\left(X_{0}^{q}\right)<$ $\infty$ and the moment conditions (2.7) hold with some $q \in \mathbb{N}$. Suppose that $\widetilde{b}=0$ (hence the process is critical). Then

$$
\sup _{t \in \mathbb{R}_{+}} \frac{\mathbb{E}\left(X_{t}^{q}\right)}{(1+t)^{q}}<\infty
$$

In particular, $\mathbb{E}\left(X_{t}^{q}\right)=\mathrm{O}\left(t^{q}\right)$ as $t \rightarrow \infty$ in the sense that $\lim \sup _{t \rightarrow \infty} t^{-q} \mathbb{E}\left(X_{t}^{q}\right)<\infty$.

B.2 Lemma. Let $\left(X_{t}\right)_{t \in \mathbb{R}_{+}}$be a $C B I$ process with parameters $(c, \beta, b, \nu, \mu)$ such that $\mathbb{E}\left(X_{0}^{q}\right)<$ $\infty$ and the moment conditions (2.7) hold, where $q=2 p$ with some $p \in \mathbb{N}$. Suppose that $\widetilde{b}=0$ (hence the process is critical). Then, for the martingale differences $M_{n}=X_{n}-\mathbb{E}\left(X_{n} \mid X_{n-1}\right)$, $n \in \mathbb{N}$, we have $\mathbb{E}\left(M_{n}^{2 p}\right)=\mathrm{O}\left(n^{p}\right)$ as $n \rightarrow \infty$ that is, $\sup _{n \in \mathbb{N}} n^{-p} \mathbb{E}\left(M_{n}^{2 p}\right)<\infty$. 
We have $\operatorname{Var}\left(M_{k} \mid \mathcal{F}_{k-1}\right)=\operatorname{Var}\left(X_{k} \mid X_{k-1}\right)$ and $\operatorname{Var}\left(X_{k} \mid X_{k-1}=x\right)=\operatorname{Var}\left(X_{1} \mid X_{0}=x\right)$ for all $x \in \mathbb{R}_{+}$, since $\left(X_{t}\right)_{t \in \mathbb{R}_{+}}$is a time-homogeneous Markov process. Hence Proposition 4.8 in Barczy et al. [6] implies the following formula for $\operatorname{Var}\left(M_{k} \mid \mathcal{F}_{k-1}\right)$.

B.3 Proposition. Let $\left(X_{t}\right)_{t \in \mathbb{R}_{+}}$be a CBI process with parameters $(c, \beta, b, \nu, \mu)$ such that $\mathbb{E}\left(X_{0}^{2}\right)<\infty$ and the moment conditions (2.7) hold with $q=2$. Then for all $k \in \mathbb{N}$, we have

$$
\operatorname{Var}\left(M_{k} \mid \mathcal{F}_{k-1}\right)=V X_{k-1}+V_{0}
$$

where

$$
\begin{aligned}
V & :=C \int_{0}^{1} \mathrm{e}^{\widetilde{b}(1+u)} \mathrm{d} u \\
V_{0} & :=\int_{0}^{\infty} z^{2} \nu(\mathrm{d} z) \int_{0}^{1} \mathrm{e}^{2 \widetilde{b} u} \mathrm{~d} u+\widetilde{\beta} C \int_{0}^{1}\left(\int_{0}^{1-u} \mathrm{e}^{\widetilde{b} v} \mathrm{~d} v\right) \mathrm{e}^{2 \widetilde{b} u} \mathrm{~d} u .
\end{aligned}
$$

Note that $V_{0}=\operatorname{Var}\left(X_{1} \mid X_{0}=0\right)$. Moreover, if $\widetilde{b}=0$, i.e., in the critical case, we have $V=C$.

B.4 Proposition. Let $\left(X_{t}\right)_{t \in \mathbb{R}_{+}}$be a CBI process with parameters $(c, \beta, b, \nu, \mu)$ such that $\mathbb{E}\left(X_{0}^{q}\right)<\infty$ and the moment conditions (2.7) hold with some $q \in \mathbb{N}$. Then for all $j \in$ $\{1, \ldots, q\}$, there exists a polynomial $P_{j}: \mathbb{R} \rightarrow \mathbb{R}$ having degree at most $\lfloor j / 2\rfloor$, such that

$$
\mathbb{E}\left(M_{k}^{j} \mid \mathcal{F}_{k-1}\right)=P_{j}\left(X_{k-1}\right), \quad k \in \mathbb{N} .
$$

The coefficients of the polynomial $P_{j}$ depends on $c, \beta, b, \nu, \mu$.

Proof. We have

$$
\mathbb{E}\left(M_{k}^{j} \mid \mathcal{F}_{k-1}\right)=\mathbb{E}\left[\left(X_{k}-\mathbb{E}\left(X_{k} \mid X_{k-1}\right)\right)^{j} \mid X_{k-1}\right]
$$

and

$$
\mathbb{E}\left[\left(X_{k}-\mathbb{E}\left(X_{k} \mid X_{k-1}\right)\right)^{j} \mid X_{k-1}=x\right]=\mathbb{E}\left[\left(X_{1}-\mathbb{E}\left(X_{1} \mid X_{0}=x\right)\right)^{j} \mid X_{0}=x\right]
$$

for all $x \in \mathbb{R}_{+}$, since $\left(X_{t}\right)_{t \in \mathbb{R}_{+}}$is a time-homogeneous Markov process. Replacing $w$ by $\mathrm{e}^{\tilde{b} t}$ in the formula for $\mathbb{E}\left[\left(w \mathrm{e}^{-\widetilde{b} t}\left(Y_{t}-\mathbb{E}\left(Y_{t}\right)\right)^{k}\right]\right.$ in the proof of Barczy et al. [6, Theorem 4.5], and then using the law of total probability, one obtains

$$
\begin{aligned}
\mathbb{E}\left[\left(X_{t}-\mathbb{E}\left(X_{t}\right)\right)^{j}\right]= & j(j-1) c \int_{0}^{t} \mathrm{e}^{j \widetilde{b}(t-s)} \mathbb{E}\left[\left(X_{s}-\mathbb{E}\left(X_{s}\right)\right)^{j-2} X_{s}\right] \mathrm{d} s \\
& +\sum_{\ell=0}^{j-2}\left(\begin{array}{l}
j \\
\ell
\end{array}\right) \int_{0}^{\infty} z^{j-\ell} \mu(\mathrm{d} z) \int_{0}^{t} \mathrm{e}^{j \widetilde{b}(t-s)} \mathbb{E}\left[\left(X_{s}-\mathbb{E}\left(X_{s}\right)\right)^{\ell} X_{s}\right] \mathrm{d} s \\
& +\sum_{\ell=0}^{j-2}\left(\begin{array}{l}
j \\
\ell
\end{array}\right) \int_{0}^{\infty} z^{j-\ell} \nu(\mathrm{d} z) \int_{0}^{t} \mathrm{e}^{j \widetilde{b}(t-s)} \mathbb{E}\left[\left(X_{s}-\mathbb{E}\left(X_{s}\right)\right)^{\ell}\right] \mathrm{d} s
\end{aligned}
$$


for all $t \in \mathbb{R}_{+}$and $j \in\{1, \ldots, q\}$, and hence, for each $t \in \mathbb{R}_{+}$and $j \in\{1, \ldots, q\}$, there exists a polynomial $P_{t, j}: \mathbb{R} \rightarrow \mathbb{R}$ having degree at most $\lfloor j / 2\rfloor$, such that

$$
\mathbb{E}\left[\left(X_{t}-\mathbb{E}\left(X_{t}\right)\right)^{j}\right]=\mathbb{E}\left[P_{t, j}\left(X_{0}\right)\right]
$$

where the coefficients of the polynomial $P_{t, j}$ depends on $c, \beta, b, \nu, \mu$, which clearly implies the statement with $P_{j}:=P_{1, j}$.

B.5 Corollary. Let $\left(X_{t}\right)_{t \in \mathbb{R}_{+}}$be a CBI process with parameters $(c, \beta, b, \nu, \mu)$ such that $X_{0}=0, \beta \neq 0$ or $\nu \neq 0$, and $\widetilde{b}=0$ (hence the process is critical). Suppose that the moment conditions (2.7) hold with some $q \in \mathbb{N}$. Then

$$
\mathbb{E}\left(X_{k}^{i}\right)=\mathrm{O}\left(k^{i}\right), \quad \mathbb{E}\left(M_{k}^{2 j}\right)=\mathrm{O}\left(k^{j}\right)
$$

for $i, j \in \mathbb{Z}_{+}$with $i \leqslant q$ and $2 j \leqslant q$.

If, in addition, $C=0$, then

$$
\mathbb{E}\left(\left|M_{k}\right|^{i}\right)=\mathrm{O}(1)
$$

for $i \in \mathbb{Z}_{+}$with $i \leqslant q$.

Proof. The first and second statements follow from Lemmas B.1 and B.2, respectively.

If $C=0$, then, by Lemma $\mathrm{A.2}, M_{k}, k \in \mathbb{N}$, are independent and identically distributed, thus

$$
\mathbb{E}\left(\left|M_{k}\right|^{i}\right)=\mathbb{E}\left(\left|M_{1}\right|^{i}\right)=\mathrm{O}(1)
$$

for $i \in \mathbb{Z}_{+}$with $i \leqslant q$.

B.6 Corollary. Let $\left(X_{t}\right)_{t \in \mathbb{R}_{+}}$be a CBI process with parameters $(c, \beta, b, \nu, \mu)$ such that $X_{0}=0, \beta \neq 0$ or $\nu \neq 0$, and $\widetilde{b}=0$ (hence the process is critical). Suppose that the moment conditions (2.7) hold with some $\ell \in \mathbb{N}$. Then

(i) for all $i \in \mathbb{Z}_{+}$with $i \leqslant\lfloor\ell / 2\rfloor$, and for all $\theta>i+1$, we have

$$
n^{-\theta} \sum_{k=1}^{n} X_{k}^{i} \stackrel{\mathbb{P}}{\longrightarrow} 0 \quad \text { as } n \rightarrow \infty
$$

(ii) for all $i \in \mathbb{Z}_{+}$with $i \leqslant \ell$, for all $T>0$, and for all $\theta>i+\frac{i}{\ell}$, we have

$$
n^{-\theta} \sup _{t \in[0, T]} X_{\lfloor n t\rfloor}^{i} \stackrel{\mathbb{P}}{\longrightarrow} 0 \quad \text { as } n \rightarrow \infty
$$

(iii) for all $i \in \mathbb{Z}_{+}$with $i \leqslant\lfloor\ell / 4\rfloor$, for all $T>0$, and for all $\theta>i+\frac{1}{2}$, we have

$$
n^{-\theta} \sup _{t \in[0, T]}\left|\sum_{k=1}^{\lfloor n t\rfloor}\left[X_{k}^{i}-\mathbb{E}\left(X_{k}^{i} \mid \mathcal{F}_{k-1}\right)\right]\right| \stackrel{\mathbb{P}}{\longrightarrow} 0 \quad \text { as } n \rightarrow \infty \text {. }
$$

Proof. The statements can be derived exactly as in Barczy et al. 4, Corollary 9.2 of arXiv version]. 


\section{CLS estimators}

C.1 Lemma. If $\left(X_{t}\right)_{t \in \mathbb{R}_{+}}$is a CBI process with parameters $(c, \beta, b, \nu, \mu)$ such that $\widetilde{b}=0$ (hence it is critical), $\mathbb{E}\left(X_{0}\right)<\infty$, and the moment condition (2.3) holds, then $\mathbb{P}\left(H_{n}\right) \rightarrow 1$ as $n \rightarrow \infty$, and hence, the probability of the existence of a unique CLS estimator $\left(\widehat{\varrho}_{n}, \widehat{\bar{\beta}}_{n}\right)$ converges to 1 as $n \rightarrow \infty$, and this CLS estimator has the form given in (3.5) on the event $H_{n}$.

Proof. First, note that for all $n \in \mathbb{N}$,

$$
\begin{aligned}
\Omega \backslash H_{n} & =\left\{\omega \in \Omega: \sum_{k=1}^{n} X_{k-1}^{2}(\omega)-\frac{1}{n}\left(\sum_{i=1}^{n} X_{i-1}(\omega)\right)^{2}=0\right\} \\
& =\left\{\omega \in \Omega: \sum_{k=1}^{n}\left(X_{k-1}(\omega)-\frac{1}{n} \sum_{i=1}^{n} X_{i-1}(\omega)\right)^{2}=0\right\} \\
& =\left\{\omega \in \Omega: X_{k-1}(\omega)=\frac{1}{n} \sum_{i=1}^{n} X_{i-1}(\omega), k \in\{1, \ldots, n\}\right\} \\
& =\left\{\omega \in \Omega: 0=X_{0}(\omega)=X_{1}(\omega)=\cdots=X_{n-1}(\omega)\right\} \\
& =\left\{\omega \in \Omega: \frac{1}{n^{2}} \sum_{i=1}^{n} X_{i-1}(\omega)=0\right\},
\end{aligned}
$$

where we used that $X_{0}=0$ and $X_{k} \geqslant 0, k \in \mathbb{Z}_{+}$.

By continuous mapping theorem, we obtain

$$
\frac{1}{n^{2}} \sum_{k=1}^{n} X_{k} \stackrel{\mathcal{D}}{\longrightarrow} \int_{0}^{1} \mathcal{Y}_{t} \mathrm{~d} t \quad \text { as } n \rightarrow \infty,
$$

see, e.g., the method of the proof of Proposition 3.1 in Barczy et al. [3].

By the proof of Theorem 3.4, we have $\mathbb{P}\left(\int_{0}^{1} \mathcal{Y}_{t} \mathrm{~d} t>0\right)=1$. Thus the distribution function of $\int_{0}^{1} \mathcal{Y}_{t} \mathrm{~d} t$ is continuous at 0 , and hence, by (C.1),

$$
\mathbb{P}\left(H_{n}\right)=\mathbb{P}\left(\sum_{i=1}^{n} X_{i-1}>0\right)=\mathbb{P}\left(\frac{1}{(n-1)^{2}} \sum_{i=1}^{n} X_{i-1}>0\right) \rightarrow \mathbb{P}\left(\int_{0}^{1} \mathcal{Y}_{t} \mathrm{~d} t>0\right)=1
$$

as $n \rightarrow \infty$.

\section{A version of the continuous mapping theorem}

The following version of continuous mapping theorem can be found for example in Kallenberg [15, Theorem 3.27]. 
D.1 Lemma. Let $\left(S, d_{S}\right)$ and $\left(T, d_{T}\right)$ be metric spaces and $\left(\xi_{n}\right)_{n \in \mathbb{N}}$, $\xi$ be random elements with values in $S$ such that $\xi_{n} \stackrel{\mathcal{D}}{\longrightarrow} \xi$ as $n \rightarrow \infty$. Let $f: S \rightarrow T$ and $f_{n}: S \rightarrow T, n \in \mathbb{N}$, be measurable mappings and $C \in \mathcal{B}(S)$ such that $\mathbb{P}(\xi \in C)=1$ and $\lim _{n \rightarrow \infty} d_{T}\left(f_{n}\left(s_{n}\right), f(s)\right)=0$ if $\lim _{n \rightarrow \infty} d_{S}\left(s_{n}, s\right)=0$ and $s \in C$. Then $f_{n}\left(\xi_{n}\right) \stackrel{\mathcal{D}}{\longrightarrow} f(\xi)$ as $n \rightarrow \infty$.

\section{E Convergence of random step processes}

We recall a result about convergence of random step processes towards a diffusion process, see Ispány and Pap [12. This result is used for the proof of convergence (4.1).

E.1 Theorem. Let $\gamma: \mathbb{R}_{+} \times \mathbb{R}^{d} \rightarrow \mathbb{R}^{d \times r}$ be a continuous function. Assume that uniqueness in the sense of probability law holds for the SDE

$$
\mathrm{d} \mathcal{U}_{t}=\gamma\left(t, \mathcal{U}_{t}\right) \mathrm{d} \mathcal{W}_{t}, \quad t \in \mathbb{R}_{+}
$$

with initial value $\mathcal{U}_{0}=\boldsymbol{u}_{0}$ for all $\boldsymbol{u}_{0} \in \mathbb{R}^{d}$, where $\left(\mathcal{W}_{t}\right)_{t \in \mathbb{R}_{+}}$is an r-dimensional standard Wiener process. Let $\left(\mathcal{U}_{t}\right)_{t \in \mathbb{R}_{+}}$be a solution of (E.1) with initial value $\mathcal{U}_{0}=\mathbf{0} \in \mathbb{R}^{d}$.

For each $n \in \mathbb{N}$, let $\left(\boldsymbol{U}_{k}^{(n)}\right)_{k \in \mathbb{N}}$ be a sequence of d-dimensional martingale differences with respect to a filtration $\left(\mathcal{F}_{k}^{(n)}\right)_{k \in \mathbb{Z}_{+}}$, that is, $\mathbb{E}\left(\boldsymbol{U}_{k}^{(n)} \mid \mathcal{F}_{k-1}^{(n)}\right)=0, n \in \mathbb{N}, k \in \mathbb{N}$. Let

$$
\mathcal{U}_{t}^{(n)}:=\sum_{k=1}^{\lfloor n t\rfloor} \boldsymbol{U}_{k}^{(n)}, \quad t \in \mathbb{R}_{+}, \quad n \in \mathbb{N} .
$$

Suppose that $\mathbb{E}\left(\left\|\boldsymbol{U}_{k}^{(n)}\right\|^{2}\right)<\infty$ for all $n, k \in \mathbb{N}$. Suppose that for each $T>0$,

(i) $\sup _{t \in[0, T]}\left\|\sum_{k=1}^{\lfloor n t\rfloor} \operatorname{Var}\left(\boldsymbol{U}_{k}^{(n)} \mid \mathcal{F}_{k-1}^{(n)}\right)-\int_{0}^{t} \boldsymbol{\gamma}\left(s, \mathcal{U}_{s}^{(n)}\right) \boldsymbol{\gamma}\left(s, \mathcal{U}_{s}^{(n)}\right)^{\top} \mathrm{d} s\right\| \stackrel{\mathbb{P}}{\longrightarrow} 0$,

(ii) $\sum_{k=1}^{\lfloor n T\rfloor} \mathbb{E}\left(\left\|\boldsymbol{U}_{k}^{(n)}\right\|^{2} \mathbb{1}_{\left\{\left\|\boldsymbol{U}_{k}^{(n)}\right\|>\theta\right\}} \mid \mathcal{F}_{k-1}^{(n)}\right) \stackrel{\mathbb{P}}{\longrightarrow} 0$ for all $\theta>0$

where $\stackrel{\mathbb{P}}{\longrightarrow}$ denotes convergence in probability. Then $\boldsymbol{U}^{(n)} \stackrel{\mathcal{D}}{\longrightarrow} \mathcal{U}$ as $n \rightarrow \infty$.

Note that in (i) of Theorem E.1, $\|\cdot\|$ denotes a matrix norm, while in (ii) it denotes a vector norm.

\section{References}

[1] Barczy, M., Döring, L., Li, Z. and PAP, G. (2013). On parameter estimation for critical affine processes. Electronic Journal of Statistics 7 647-696. 
[2] Barczy, M., Ispány, M. and PAP, G. (2011). Asymptotic behavior of unstable INAR $(p)$ processes. Stochastic Processes and their Applications 121(3) 583-608.

[3] Barczy, M., Ispány, M. and PAP, G. (2010). Asymptotic behavior of CLS estimators of autoregressive parameter for nonprimitive unstable INAR(2) models. Available on the ArXiv: http://arxiv.org/abs/1006.4641.

[4] Barczy, M., Ispány, M. and PAp, G. (2014). Asymptotic behavior of conditional least squares estimators for unstable integer-valued autoregressive models of order 2. Scandinavian Journal of Statistics 41(4) 866-892.

[5] Barczy, M., Li, Z. and PAP, G. (2014). Stochastic differential equation with jumps for multi-type continuous state and continuous time branching processes with immigration. To appear in ALEA. Latin American Journal of Probability and Mathematical Statistics. Available on ArXiv: http://arxiv.org/abs/1403.0245

[6] Barczy, M., Li, Z. and PAP, G. (2014). Moment formulas for multi-type continuous state and continuous time branching processes with immigration. To appear in Journal of Theoretical Probability. Available on ArXiv: http://arxiv.org/abs/1404.0636

[7] Barczy, M. and PAP, G. (2014). Asymptotic behavior of critical irreducible multi-type continuous state and continuous time branching processes with immigration. Available on ArXiv: http://arxiv.org/abs/1404.2242

[8] Dawson, D. A. and Li, Z. (2006). Skew convolution semigroups and affine Markov processes. The Annals of Probability 34(3) 1103-1142.

[9] Duffie, D., Filipović, D. and Schachermayer, W. (2003). Affine processes and applications in finance. Annals of Applied Probability 13 984-1053.

[10] Huang, J., Ma, C. and Zhu, C. (2011). Estimation for discretely observed continuous state branching processes with immigration. Statistics and Probability Letters 81 11041111.

[11] Ikeda, N. and Watanabe, S. (1989). Stochastic Differential Equations and Diffusion Processes, 2nd ed. North-Holland/Kodansha, Amsterdam/Tokyo.

[12] Ispány, M. and PAP, G. (2010). A note on weak convergence of step processes. Acta Mathematica Hungarica 126(4) 381-395.

[13] Ispány, M., KÖRmendi, K. and PAP, G. (2014). Asymptotic behavior of CLS estimators for 2-type doubly symmetric critical Galton-Watson processes with immigration. Bernoulli 20(4) 2247-2277.

[14] Jacod, J. and Shiryaev, A. N. (2003). Limit Theorems for Stochastic Processes, 2nd ed. Springer-Verlag, Berlin. 
[15] Kallenberg, O. (1997). Foundations of Modern Probability. Springer, New York, Berlin, Heidelberg.

[16] Kyprianou, A. E. (2014) Fluctuations of Lévy processes with applications, 2nd ed. Springer, Heidelberg.

[17] Li, Z. (2011). Measure-Valued Branching Markov Processes. Springer-Verlag, Heidelberg.

[18] Li, Z. and MA, C. (2013). Asymptotic properties of estimators in a stable Cox-IngersollRoss model. Available on the ArXiv: http://arxiv.org/abs/1301.3243

[19] Overbeck, L. and Rydén, T. (1997). Estimation in the Cox-Ingersoll-Ross model. Econometric Theory 13(3) 430-461.

[20] Revuz,D. and Yor, M. (2001). Continuous Martingales and Brownian Motion, 3rd ed., corrected 2nd printing. Springer-Verlag, Berlin.

[21] Wei, C. Z. and Winnicki, J. (1989). Some asymptotic results for the branching process with immigration. Stochastic Processes and their Applications 31(2) 261-282.

[22] Wei, C. Z. and Winnicki, J. (1990). Estimation of the means in the branching process with immigration. The Annals of Statistics 18 1757-1773. 